\title{
The Role of GABAergic Inhibition in Processing of Interaural Time Difference in the Owl's Auditory System
}

\author{
Ichiro Fujita and Masakazu Konishi \\ Division of Biology, California Institute of Technology, Pasadena, California 91125
}

The barn owl uses interaural time differences (ITDs) to localize the azimuthal position of sound. ITDs are processed by an anatomically distinct pathway in the brainstem. Neuronal selectivity for ITD is generated in the nucleus laminaris (NL) and conveyed to both the anterior portion of the ventral nucleus of the lateral lemniscus (VLVa) and the central (ICc) and external (ICX) nuclei of the inferior colliculus. With tonal stimuli, neurons in all regions are found to respond maximally not only to the real ITD, but also to ITDs that differ by integer multiples of the tonal period. This phenomenon, phase ambiguity, does not occur when ICX neurons are stimulated with noise.

The main aim of this study was to determine the role of GABAergic inhibition in the processing of ITDs. Selectivity for ITD is similar in the NL and VLVa and improves in the ICc and ICX. lontophoresis of bicuculline methiodide (BMI), a selective $\mathrm{GABA}_{\mathrm{A}}$ antagonist, decreased the ITD selectivity of ICC and ICX neurons, but did not affect that of VLVa neurons. Responses of VLVa and ICc neurons to unfavorable ITDs were below the monaural response levels. BMI raised both binaural responses to unfavorable ITDs and monaural responses, though the former remained smaller than the latter. During BMI application, ICx neurons showed phase ambiguity to noise stimuli and no longer responded to a unique ITD. BMI increased the response magnitude and changed the temporal discharge patterns in the VLVa, ICc, and ICx. Iontophoretically applied GABA exerted effects opposite to those of BMI, and the effects could be antagonized with simultaneous application of BMI. These results suggest that GABAergic inhibition (1) sharpens ITD selectivity in the ICC and ICX, (2) contributes to the elimination of phase ambiguity in the $\mathrm{ICX}$, and (3) controls response magnitude and temporal characteristics in the VLVa, ICc, and ICX. Through these actions, GABAergic inhibition shapes the horizontal dimension of the auditory receptive fields.

The barn owl uses interaural differences in time (ITDs) and intensity (IIDs) to locate the horizontal and vertical positions of sound, respectively (Moiseff and Konishi, 1981, 1989a,b).

\footnotetext{
Received July 26, 1990; revised Oct. 9, 1990; accepted Oct. 17, 1990.

We thank Terry Takahashi, Ralph Adolphs, and Susan Volman for their help throughout the work; Caroly Shumway, Hermann Wagner, Larry Proctor, Catherine Carr, and Lennie Maler for comments on the manuscript; Jack Wathey, Hermann Wagner, and Larry Proctor for help with computer programs; Michael Paulin and Koichi Mori for help with statistical analysis; and Masashi Kawasaki for technical advice on electrodes. This work was supported by a grant from the Uehara Memorial Foundation to I.F. and by NIH Grant NS14617 to M.K.

Correspondence should be addressed to Ichiro Fujita, Ph.D., Laboratory for Neural Information Processing, Frontier Research Program, RIKEN Institute, Hirosawa 2-1, Wako, Saitama 351-01, Japan.

Copyright $\odot 1991$ Society for Neuroscience $0270-6474 / 91 / 110722-18 \$ 03.00 / 0$
}

The owl's auditory system processes ITD and IID in anatomically and physiologically separate pathways (Konishi, 1986; Konishi et al., 1988). Neuronal selectivity for ITD emerges in the nucleus laminaris (NL) and is carried to higher nuclei, the anterior portion of the ventral nucleus of the lateral lemniscus (VLVa) and a subdivision ("core") of the central nucleus of the inferior colliculus (ICc; Fig. 1). The ICc core projects ipsilaterally to the external nucleus of the inferior colliculus (ICx; Knudsen, 1984; Wagner et al., 1987) and to the contralateral ICc "lateral shell," which, in turn, projects to the ICx on the same side (Fig. 1; Takahashi et al., 1989). ICc neurons are more selective for ITD than NL and VLVa neurons, and ICx neurons are more sharply tuned to ITD than ICc neurons (present results; see Figs. $2,3)$. Neurons in the NL, VLVa, and ICc respond maximally to 1 ITD and its phase equivalents, whether the stimulus is a tone or noise. This phenomenon is termed "phase ambiguity." ICx neurons, however, do not show phase ambiguity to noise stimuli (Takahashi and Konishi, 1986).

Evidence suggests that the elimination of phase ambiguity in the ICx involves inhibition (Takahashi and Konishi, 1986). Inhibition is also implicated in generating the center-surround receptive fields of ICx neurons (Knudsen and Konishi, 1978b). An immunocytochemical study has shown that all nuclei in the barn owl's auditory brainstem and inferior colliculus contain GABAergic cell bodies, terminals, or both (Carr et al., 1989). Because neuronal selectivity for ITD can be traced from the first site of its emergence (NL) to the highest station (ICX) where the neuronal map of auditory space is formed, the "ITD pathway" of the barn owl auditory system provides unique opportunities to study the contributions of inhibition to a stimulus selectivity at successive stages of its synthesis. In the present study, we applied bicuculline methiodide (BMI; a selective antagonist of GABA) or GABA by microiontophoresis into 1 of the 4 sites, the immediate vicinity of neurons in the VLVa, the 2 subdivisions of ICc (core and lateral shell), or ICx, while examining ITD selectivity of these neurons. We present evidence for the contribution of GABA-mediated inhibition to the improvement of ITD selectivity, the elimination of phase ambiguity, and other response properties.

Part of this study has been reported in abstract form (Fujita and Konishi, 1988, 1989a).

\section{Materials and Methods}

We will briefly describe those procedures that have already been published (Takahashi and Konishi, 1986; Wagner et al., 1987) and will describe those new to this paper in detail.

Surgery and animal care. Barn owls (Tyto alba) of either sex $(n=$ 10 ), weighing $403-542 \mathrm{gm}$, were anesthetized with intramuscular injections of ketamine hydrochloride $(9.8-15.4 \mathrm{mg} / \mathrm{kg} / \mathrm{hr})$. Each bird's head was fixed in a custom-made stereotaxic device with ear bars and 
a beak bar, as described previously (Takahashi and Konishi, 1986). After topical application of local anesthetics (lidocaine), the scalp was cut, and a stainless-steel headplate was cemented to the skull. Recording experiments started 1-4 d after this surgery. Each bird was anesthetized as before, and the head was held by bolting the headplate to the stereotaxic apparatus. A small hole was then made in the skull, the dura mater was incised and reflected, and electrodes (see below) were inserted into the brain. The owl was anesthetized with ketamine hydrochloride (dose as given above), and its body temperature was maintained at $40^{\circ} \mathrm{C}$ by a heating pad. When a bird was allowed to survive after an experiment, the opening in the skull was sealed with dental cement. An antibiotic ointment and a local anesthetic were applied topically to the skin incision, which was then sutured. Each bird was kept in a separate cage and inspected daily. Birds that did not resume feeding within 48 $\mathrm{hr}$ after surgery received injections of $5 \%$ glucose in saline $(5-10 \mathrm{ml} / \mathrm{d})$ until they began to eat normally.

Recording and drug application. Extracellular recordings and drug ejections were made with triple-barreled glass micropipettes. The combined tip of the electrode assemblies was broken to an outer diameter of $6-12 \mu \mathrm{m}$. The recording electrode barrel was filled with Wood's metal alloy or indium and plated with gold and platinum (1-9 M $\Omega$ at $1 \mathrm{kHz}$ AC; Frank and Becker, 1964). The second barrel contained BMI (Sigma; $5 \mathrm{~mm}$ in $0.9 \% \mathrm{NaCl} ; \mathrm{pH}, 3.2-3.5)$. The third barrel contained either GABA (Sigma; $0.5 \mathrm{M}$ in $0.9 \% \mathrm{NaCl}$; $\mathrm{pH}, 3.5)$ or vehicle $(0.9 \% \mathrm{NaCl}$; $\mathrm{pH}, 3.2-3.5$ ). The $\mathrm{pH}$ of solutions was adjusted with $\mathrm{HCl}$. The concentration of GABA and BMI is standard in previous iontophoretic studies (e.g., Sillito, 1975; Dykes et al., 1984; Caspary et al., 1985; Müller and Scheich, 1988). The reason that GABA has been used at a higher concentration is probably related to the presence of high-aflinity uptake mechanisms for GABA, but not for BMI, in the brain (Iversen and Bloom, 1972). Solutions were freshly made before each experiment, and pipettes were filled immediately before use. The electrode resistance of these drug barrels was 10-40 M . This method allowed us to obtain single units with high signal-to-noise ratio and often hold the units more than $1 \mathrm{hr}$ (see Figs. 4B, 17B) while ejecting drugs effectively from another barrel. Retaining currents of -10 to $-15 \mathrm{nA}$ were employed between drug applications to minimize spontaneous leakage of drugs from micropipettes. Current balancing was used to decrease possible current effects; while ejection current was applied to one barrel, current of equal amount and opposite polarity was passed through the other pipette. This procedure, however, turned out to be unnecessary for the lowintensity current we used.

Stimulation. All experiments were conducted in a sound-attenuated room. Auditory stimuli of $100-\mathrm{msec}$ bursts $(5-\mathrm{msec}$ rise/fall time) of either noise or tone were delivered at a rate of $1.0-1.2 / \mathrm{sec}$ through earphones inserted to the owl's external ear canal. The sound level was adjusted to $10-30 \mathrm{~dB}$ (usually $20 \mathrm{~dB}$ ) above the neuron's threshold. A digital delay circuit (Moiseff and Konishi, 1981) and digital attenuators controlled ITD and IID independently. When a noise burst was used for the measurement of neuronal selectivity for ITD, the same noise was delivered to the 2 ears, with the exception that the noise waveform was time shifted in one ear with respect to the other ear. A Fourier analysis of the original noise and its time-shifted version showed the same power and phase spectra, but the phase of all frequency components in the time-shifted version was shifted by an angle equivalent to the ITD. Sampling range was $\pm 300 \mu \mathrm{sec}$ for ITD and $\pm 50 \mathrm{~dB}$ for IID. Sampling interval was $30 \mu \mathrm{sec}$ for ITD and $5 \mathrm{~dB}$ for IID. In our convention, positive ITDs indicate that the stimulus in the right ear led, and negative ITDs indicate that the stimulus in the left ear led. Positive and negative IIDs indicate that the stimulus was louder in the right and left ear, respectively. Calibration and performance of the sound delivery system have been described previously (Takahashi and Konishi, 1986).

Data acquisition. The number of spikes was counted during the 120 msec after the stimulus onset and was averaged over 5 stimulus repetitions. Data were collected and analyzed on line with a computer (PDP11/40) and stored on disks for subsequent analyses. Responses to each ITD were plotted as either the actual number of spikes (non-normalized plots) or the rclative level (\%) of the maximal response (normalized plots). The resulting curve is referred to as an "ITD curve." An ITD curve shows cyclic changes in spike rate as a function of ITD. The segments of the curve where maximal and minimal discharges occur are termed "peak" and "trough." As a measure of ITD selectivity, we used the width of the peak at the midpoint between the maximum and the minimum of the curve ("half-peak width"; cf. Yin and (han, 1988). For the measurement of half-peak widths, we chose the peak at that

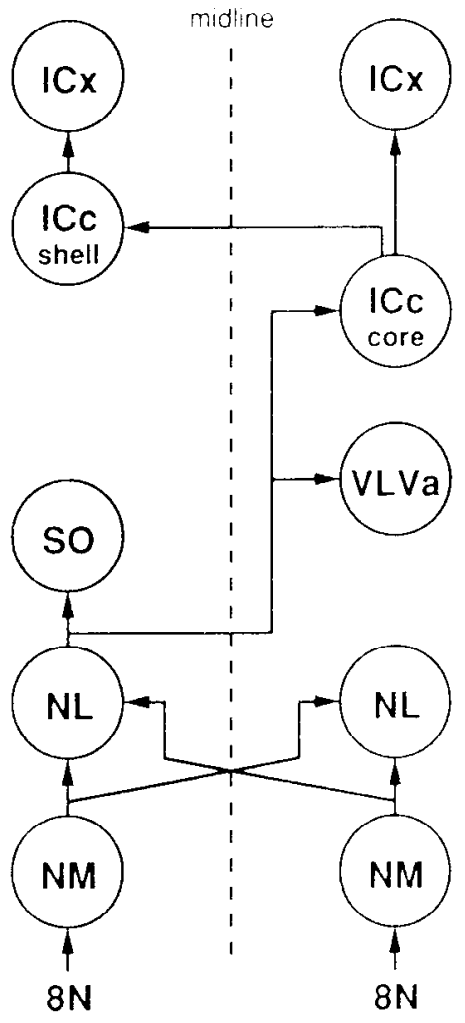

Figure 1. Nuclei and connections for processing ITD in the barn owl ("ITD pathway"; modified from Takahashi and Konishi, 1988b). For simplicity, projections beyond NL are shown only on 1 side. NL is the first site of binaural convergence of temporal information, where neuronal selectivity for ITD is generated. NL projects to VLVa and ICC core. The core projects ipsilaterally to ICx (Knudesen, 1984; Wagner et al., 1987) and contralaterally to the lateral part of the ICc shell (Takahashi et al., 1989). The ICc lateral shell, in turn, projects to ICX on the same side. $8 N$, auditory nerve; $S O$, superior olivary nucleus.

ITD where the primary peak occurred with noise stimuli in the ICx, at the "array-specific" ITD in the ICc (Wagner et al., 1987; see below), or at the ITD nearest to $0 \mu \mathrm{sec}$ in the NL and VLVa. Half-peak widths were measured on tonal ITD curves obtained from single units. We also measured the difference in the spike number per stimulus between peak and trough (trough-peak difference) and the ratio of trough to peak (trough : peak ratio) for these peaks. For the measurement of half-peak width, trough-peak difference, and trough: peak ratio, we averaged 2 troughs beside the chosen peak.

Experimental protocols. Electrodes were stereotaxically placed in the brain. When extracellular action potentials were isolated from an ITDselective neuron, the ITD that yielded the maximal response to noise was qualitatively determined by changing ITDs manually. At this ITD, we determined the frequency that evoked the maximal response (best frequency; BF) by monitoring an oscilloscope and listening to an audiomonitor (Takahashi and Konishi, 1986). Neuronal selectivity for ITDs was then quantitatively examined under computer control with both tone stimuli at BF and noise stimuli.

The ICc is tonotopically organized; lower-frequency neurons are located dorsally, and higher-frequency neurons, ventrally (Knudsen and Konishi, 1978a). An ITD maximally activates all neurons encountered at different depths in a vertical penetration, that is, all neurons with different BFs (Wagner et al., 1987). This ITD is called an "array-specific ITD." The ICc is thus composed of iso-ITD columns perpendicular to the isofrequency laminae. By obtaining more than 3 recordings at different depths at least $300 \mu \mathrm{m}$ apart, we determined the array-specific ITD for each penetration in the ICc. The previously analyzed map of array-specific ITDs in the ICc (Wagner et al., 1987) also helped us to determine the array-specific ITD for each penetration. Responses to IIDs were subsequently examined at the array-specific ITD (in the ICc) or at the most favorable ITD with noise (in the ICx). Combining all the 
Table 1. Unit recordings studied with iontophoretic application of BMI, GABA, and vehicle

\begin{tabular}{lccclc} 
& BMI & $\begin{array}{l}\text { BMI, } \\
\text { GABA }\end{array}$ & $\begin{array}{l}\text { BMI, } \\
\text { Vehicle }^{\alpha}\end{array}$ & Total & $\begin{array}{l}\text { Number } \\
\text { of owls } \\
\text { used }\end{array}$ \\
\hline VLVa & $9(7)^{h}$ & $5(3)$ & $0(0)$ & $14(10)$ & 3 \\
ICc core & $8(3)$ & $6(4)$ & $10(5)$ & $24(12)$ & 6 \\
ICc lateral shell & $12(7)$ & $2(2)$ & $9(8)$ & $23(17)$ & 8 \\
ICx & $12(10)$ & $7(6)$ & $8(6)$ & $27(22)$ & 7 \\
Total & $41(27)$ & $20(15)$ & $27(19)$ & $88(61)$ & $10^{c}$ \\
\hline
\end{tabular}

Vehicle, $0.9 \% \mathrm{NaCl} ; \mathrm{pH}, 3.2-3.5$.

"Number of single units is shown in parentheses.

The same owl was used for recording from different nuclei.

results of frequency tuning, noise- and tone-ITD tuning, and IID tuning allowed us to identify the recording sites quite reliably before histology (see Results).

After we identified physiologically the nucleus to which neurons belong, their ITD selectivity was examined during and subsequent to the iontophoretic application of drugs and vehicle. Drug application was started from a low ejecting current of +5 or $+10 \mathrm{nA}$ and was increased by $10-\mathrm{nA}$ steps to $+50 \mathrm{nA}$ until effects were observed. Current of a given intensity was continuously applied for 5 min to more than $1 \mathrm{hr}$. We usually tested the drug effects at threshold current intensity. In 10 neurons, the protocol of Sillito (1975) was employed to determine an appropriate amount of BMI ejection current. We first determined the minimal GABA iontophoretic current that suppressed neural responses to favorable ITDs. We then established the minimal BMI ejection current that blocked the inhibition (see Fig. 17).

Histology. For histological verification, electrolytic lesions were made by passing current $(-2 \mu \mathrm{A}, 10 \mathrm{sec})$ at 12 recording sites in 8 owls. After a survival period of 3-7 d, birds were given ketamine and an overdose of pentobarbital (32-44 mg/kg, i.m.), transcardially exsanguinated with $0.1 \mathrm{~m}$ phosphate-buffered saline, and fixed by perfusion either with $10 \%$ formal saline or a mixture of $4 \%$ paraformaldehyde and $0.5 \%$ glutaraldehyde in $0.1 \mathrm{~m}$ phosphate buffer $(\mathrm{pH}, 7.4)$. The skull was placed in a stereotaxic device, similar to that used in the recording experiments, and the brain was blocked parallel to the plane of electrode penetrations. The brain was then removed from the skull and infiltrated with $30 \%$ sucrose for cryoprotection. Serial frozen sections were cut at $30 \mu \mathrm{m}$, collected, and stored in ice-cold $0.1 \mathrm{M}$ phosphate buffer. Every third section was stained with cresyl violet. We processed some sections from a paraformaldehyde-glutaraldehyde-fixed brain for immunocytochemistry for vitamin D-dependent calcium-binding protein (CaBP or "calbindin") in order to differentiate 2 subdivisions of the ICc, the "core" (CaBP-immunoreactive) and the "shell" (CaBP-immunonegative; Takahashi et al., 1987).

\section{Results}

Neurons analyzed

Most of the neurons were unambiguously identified by the physiological criteria (Knudsen and Konishi, 1978a; Moiseff and Konishi, 1983; Sullivan and Konishi, 1986; Takahashi and Konishi, 1986; Wagner et al., 1987; Fujita and Konishi, 1989b; Takahashi et al., 1989) and the stereotaxic coordinates known in our laboratory. Some neurons could not be classified, however, and were therefore excluded from the present analysis. Physiological criteria included phase locking, frequency tuning, tonotopic organization, ITD selectivity, and IID selectivity. NL neurons are phase locked, narrowly tuned to frequency, tonotopically organized (high frequencies are represented anteromedially, whereas low frequencies are represented posterolaterally), and ITD selective (phase ambiguous with tone or noise stimulus) and show no IID selectivity (Moiseff and Konishi, 1983; Sullivan and Konishi, 1986). VLVa neurons show no phase locking and are narrowly tuned to frequency, tonotopically organized (high-frequency neurons are found posteriorly, and low-frequency ncurons are located anteriorly), ITD selective (phase ambiguous with tone or noise), and not IID selective (Moiseff and Konishi, 1983; H. Wagner, unpublished observations). ICc core neurons show no phase locking, are narrowly tuned to frequency, tonotopically organized (low-frequency neurons are found dorsally, whereas the ventral part represents high frequencies), and ITD selective (phase ambiguous with tone or noise; represents mainly ipsilateral space), and show no IID selectivity (Wagner et al., 1987; Takahashi et al., 1989). ICc lateral-shell neurons are similar to ICc core neurons in their sharp frequency tuning, tonotopic organization, and ITD selectivity (phase ambiguous with tone or noise). However, they represent mostly contralateral space (Wagner et al., 1987; Takahashi et al., 1989) and are IID selective (Fujita and Konishi, 1989b). ICx neurons are broadly tuned to frequency and are not tonotopically organized (Knudsen and Konishi, 1978a). They are tuned to a combination of ITD and IID and arc cxclusivcly binaural (they do not respond to monaural stimulation; Moiseff and Konishi, 1983). When stimulated with noise, they do not show phase ambiguity (Takahashi and Konishi, 1986). The stereotaxic coordinates, especially depth from the brain surface, also helped us to discriminate the inferior colliculus from the VLVa and NL. Previous studies have shown that these criteria are reliable enough to identify the cells, and our own histological examination of the recording sites confirmed that neurons identified by the physiological criteria were located in the appropriate nuclei.

In the present study, we examined the effects of BMI on 61 single units and 27 multiple units. Of these recordings, 20 were also tested with $\mathrm{GABA}$ and 27 with vehicle $(0.9 \% \mathrm{NaCl} ; \mathrm{pH}$, 3.2-3.5). Table 1 shows the numbers of cells tested with drugs in each nucleus. As stated in the Materials and Methods, ITD curves of all these units were examined before, during, and after drug application. We also analyzed tonal ITD curves in another sample of 62 single units on which no drug application was made (see Fig. 3). Of these 62 units, 9 were from the NL, 4 from the VLVa, 26 from the ICc core, 18 from the ICc lateral shell, and 5 from the ICx. Eight of the 9 NL neurons are from Carr and Konishi (1990), in which recordings were made intracellularly. All figures and statistical analyses were made on data from single-unit recordings only.

\section{Neuronal selectivity for ITD without drug application}

Neurons in the NL, VLVa, ICc (both core and lateral shell), and ICx respond preferentially to discrete ranges of ITDs (Fig. 2). When these neurons are stimulated with tones, favorable and unfavorable ITDs occur cyclically; in other words, there are multiple peaks of an identical height displaced from each other by a time equal to the period of the stimulus tone. These neurons therefore do not signal a unique ITD but are "phase ambiguous" (see Figs. 4-10, 15; Moiseff and Konishi, 1983; Sullivan and Konishi, 1986; Takahashi and Konishi, 1986; Wagner et al., 1987; Fujita and Konishi, 1989b). Phase ambiguity occurs because the owl uses the difference in phase angles between the ears to derive ITD (Moiseff and Konishi, 1981), and the auditory systcm cannot discriminate between different ITDs that bring about the same interaural phase difference. Because neurons in the NL, VLVa, and ICc are sharply tuned to sound frequencies (for ICc neurons, see Knudsen, 1984), these neurons respond to the phase of the spectral components, and hence, ITD curves 


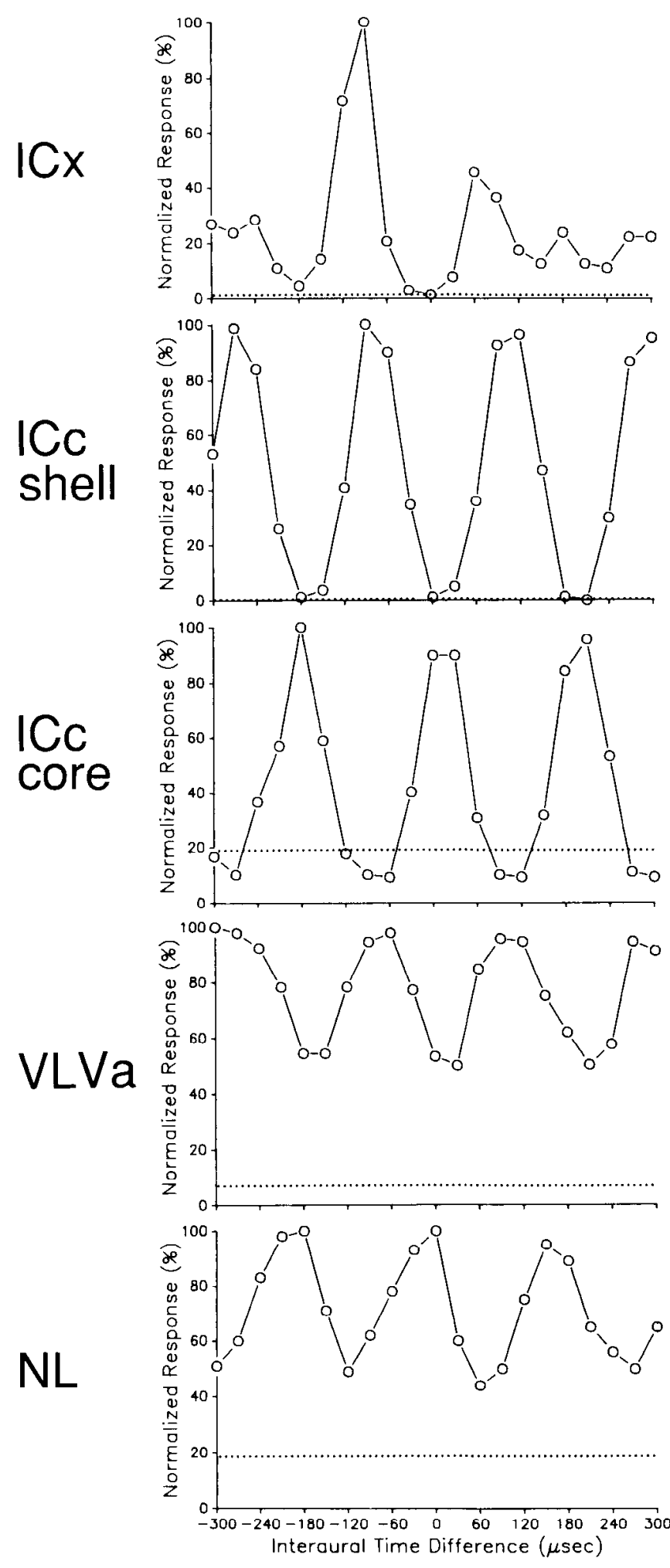

Figure 2. ITD curves obtained from neurons in 5 successive nuclei in the ITD pathway. All data in this and the following figures are from single-unit recordings. Broad-band noise was used as the stimulus. Neurons with similar BFs $(5.4-5.7 \mathrm{kHz})$ are shown. Dotted lines indicate the spontaneous firing level. Neuronal selectivity for ITD is similar between the NL and the VI.Va, hut markedly improves in the ICc core. Responses are phase ambiguous in the NL, VLVa, and ICc, but much less so in the ICx. See Figure 3 for a variety of ITD tuning within a nuclcus. Recordings were made in the left NL, ICc core, and ICc lateral shell and in the right VLVa and ICx.
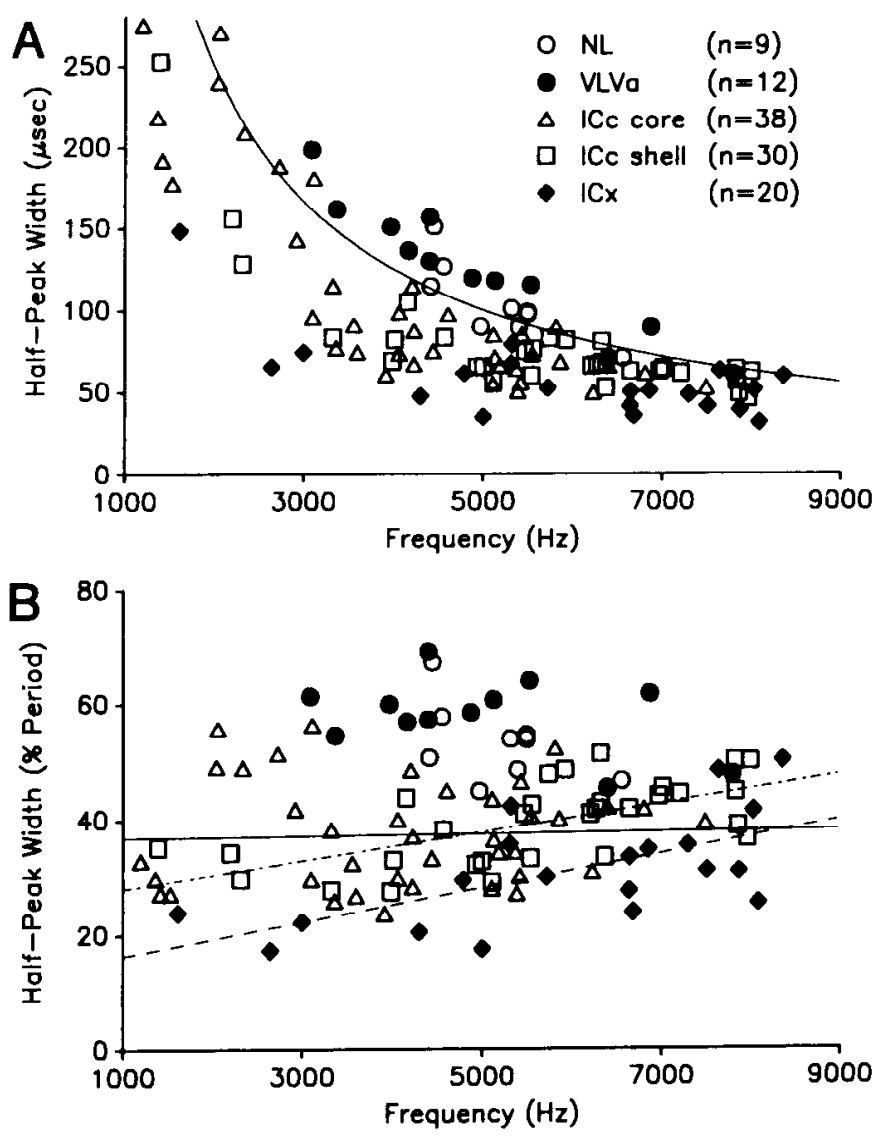

Figure 3. Half-peak width of ITD curves plotted against neuron's BF. Half-peak width $(A)$ and ratio of the width to the period of the stimulus tone $(B)$ were plotted for 109 neurons isolated from 5 successive nuclei in the ITD nathway. Tone bursts of each neuron's BF were used as stimuli. A hyperbola in $A$ is drawn through the half-value of the tonal period. Regression lines in $B$ are for the ICc core (solid), ICc lateral shell (dashed-dotted), and ICX (dashed). Data of 8 of 9 NL neurons come from Carr and Konishi (1990).

obtained with noise stimuli are essentially identical to those obtained with tonal stimuli except for the absolute level of responses (see Figs. 2, 5, 6). ICx neurons are generally more broadly tuned to frequency, if they respond to tones at all. The BFs of these neurons, however, can be determined and range usually from 5 to $8 \mathrm{kHz}$ (Knudsen and Konishi, 1978a). The ITD curves obtained with noise bursts consist of a single "primary" peak and smaller "secondary" peaks at ITDs removed from that of the primary peak by integer multiples of the period of the neuron's BF (see Figs. 2, 12; Takahashi and Konishi, 1986). At unfavorable ITDs adjacent to the primary peak, responses are weakest, often suppressed below the spontaneous firing level or totally silenced (see Figs. 2, 12A).

Figure $3 A$ shows plots of half-peak widths against BFs. Tonal ITD curves before drug application were obtained in 47 of the 61 single units in Table 1 . These 47 and another set of 62 single units tested without any drug application are the data base for Figure 3 (109 single units in total). For all nuclei, there was a clear tendency for lower-frequency neurons to have wider halfpeak widths. Distribution of half-peak widths for all nuclei roughly parallels the hyperbolic curve that results from plotting the half-value of the tonal period against frequency. If ITD curves were sinusoid, data points would lie on this hyperbola; 
any displacement of points from this hyperbola indicates deviation of ITD curves from sine curves. Most of the NL and VLVa neurons (16 of 21 units) lie above the hyperbola, while most of the inferior colliculus neurons ( 82 of 88 units) lie below it. Apparently, the half-peak width of neurons in the 2 lowerorder nuclei is wider than that of the frequency-matched inferior colliculus neurons (see also Fig. 2).

For statistical comparison, we calculated the ratio of halfpeak width to the period of each neuron's BF (\% period; Fig. $3 B)$. It ranged from 44.7 to $67.1 \%(52.9 \pm 6.4 \%$, mean $\pm \mathrm{SD}$; $n=9)$ in the $\mathrm{NL}$, from 45.2 to $69.1 \%(58.0 \pm 6.3 \% ; n=12)$ in the VLVa, from 23.6 to $56.2 \%(37.4 \pm 8.9 \% ; n=38)$ in the ICc core, from 27.6 to $51.1 \%(39.3 \pm 6.8 \% ; n=30)$ in the ICc lateral shell, and from 17.3 to $50.1 \%(31.1 \pm 9.1 \% ; n=20)$ in the ICx. A 1-way ANOVA test performed after arcsine transformation of these percentage data (Sokal and Rohlf, 1987) indicates a significant difference in the ratio of half-peak width to the period of BF among the 5 nuclei $(p<0.0001)$. A comparison between pairs of nuclei indicates no significant difference between the NL and VLVa and between the ICc core and lateral shell, while there is a difference between the ICc core and a combined sample of the NL and VLVa $(p<0.001)$ and between the ICx and a combined sample of the ICc core and lateral shell $(p<0.001)$.

We fit regression lines to data from each subdivision of the inferior colliculus (Fig. 3B). Because our sample from the NL and VLVa covered only a limited frequency range $(4.4-6.6 \mathrm{kHz}$ for the NL; $3.1-7.8 \mathrm{kHz}$ for the VLVa), we did not attempt regression analysis for these nuclei. The slopes of the regression curves for ICc lateral shell and ICx neurons were significantly steeper than that of ICc core neurons $(p<0.002$ for ICc lateral shell; $p<0.001$ for $\mathrm{ICx}$ ).

There is also a significant difference in the trough : peak ratio among the 5 nuclei $(p<0.0001,1$-way ANOVA test after arcsine transformation). The trough : peak ratio did not differ between the NL and VLVa $(0.46 \pm 0.18$, mean $\pm \mathrm{SD}$, for a combined sample; Fig. 2). Troughs became deeper in the ICc core $(0.04 \pm 0.07)$ than in the lower 2 nuclei $(p<0.001)$. The trough : peak ratio in the ICc lateral shell $(0.07 \pm 0.08)$ and $\mathrm{ICx}$ $(0.03 \pm 0.04)$ was similar to that of the ICc core. Most NL and VLVa neurons were spontaneously active, and troughs were above the spontancous discharge level in most ncurons of these nuclei (19 out of 21 neurons in our sample; see Fig. 2). By contrast, 14 of 68 neurons $(21 \%)$ in the ICc core and lateral shell did not discharge spontaneously, and the proportion of silent neurons increased up to $50 \%$ (10 of 20 neurons) in the ICx. Of 64 spontaneously active neurons in the ICc and ICx, 51 cells $(80 \%)$ reduced their responses to unfavorable ITDs below the spontaneous firing level (see Figs. 2, 4, 6-8, 10, 11).

In summary, the half-peak width was similar between the NL and VLVa, narrower in the ICc core and lateral shell, and even narrower in the ICx. Troughs were also similar in depth between the NL and VLVa and became deeper in the ICc core. Neurons in the NL, VLVa, ICc core, and ICc lateral shell were phase ambiguous with noise-burst stimulation, while ICx neurons were not. Neurons in all nuclei showed phase ambiguity with tone stimuli.

\section{General aspects of BMI effects}

Auditory responses of all units tested ( $n=88$; Table 1) were affected by iontophoresis of BMI with an ejecting current of +5 to $+50 \mathrm{nA}$. No systematic difference in the threshold current was found among different nuclei. In 10 neurons on which 2 or more intensities of ejecting currents were tested, BMI ejected with higher currents caused greater effects with a shorter delay to reach the maximum effect.

The effects of BMI became noticeable within several seconds after the start of an ejecting current, but the maximum effect was obtained only after prolonged periods of ejection (usually 10-30 min). Figure 4 shows an example of the time course of BMI effects on an ICc core neuron. When BMI was applied, responses gradually increased both to favorable and to unfavorable ITDs. The entire ITD curve gradually shifted upward in non-normalized plots (Fig. 4A). It should be noted, however, that the time course and the degree of change were not identical between peaks and troughs. Peaks and troughs reached their maxima approximately 12 min after the administration of BMI with an ejecting current of $+10 \mathrm{nA}$ (Fig. $4 B$ ). For this cell, the response magnitude recovered to its original level $7 \mathrm{~min}$ after the ejecting current was stopped. In all cells tested, complete recovery was usually obtained within 3-20 min after cessation of BMI application (see Figs. 4-6, 11, 13-15, 17).

When we used a drug barrel of low resistance ( $15 \mathrm{M} \Omega$ or lower), neuronal responses sometimes increased after the retaining current was stopped, presumably due to spontaneous leakage of BMI from the pipette. Figure $4 B$ shows such an example where only the peak of the ITD curve was raised (double arrow), but the trough remained unchanged after switching off the retaining current (see Discussion). The maximum level of this effect (double arrow) was smaller than that caused by a +10-nA ejecting current (arrow). This suggests that BMI action is dose dependent.

Because our BMI and GABA solutions were adjusted to low $\mathrm{pH}(\mathrm{pH}, 3.2-3.5)$, we examined the effects of current ejection through the vehicle-containing barrels $(0.9 \% \mathrm{NaCl} ; \mathrm{pH}, 3.2-$ 3.5 ). In all 27 recordings tested (19 single and 8 multiple units; see Table 1), neither spontaneous firing nor auditory responses were affected by current ejection through the vehicle-containing barrels, whereas BMI ejection with a current of the same amount and polarity from another barrel changed the auditory responses of these units.

In 45 of the 61 single units tested (74\%), BMI affected auditory responses, but had little or no effect on the spontaneous discharge (see Figs. 4-8, 10, 12, 15, 17). In the remaining cclls, spontaneous firing was elevated by BMI (e.g., Fig. 11). The increase in spontaneous discharge, however, cannot account for the effects of BMI on ITD curves.

\section{Effects of BMI on neuronal selectivity for ITD}

All statistical tests in this section are paired $t$ tests, and $p$ values will be shown in parentheses when the difference is significant. We present the mean and SD for the ratio of half-peak width to the period of $\mathrm{BF}$, the trough : peak ratio, and trough-peak difference. The former 2 were percentage data and were necessarily subjected to arcsine transformation before the statistical test (Sokal and Rohlf, 1987).

\section{VLVa neurons}

The administration of BMI increased VLVa neuron discharges both for favorable and for unfavorable ITDs (Fig. $5 A, B$ ). The effects were similar for the tone- (Fig. $5 A$ ) and noise-evoked (Fig. $5 B$ ) responses and were consistently observed in all recordings we examined ( $n=14$; Table 1). The BFs of these neurons ranged from 3.9 to $7.8 \mathrm{kHz}$. The administration of BMI 
did not significantly affect the half-peak width of most VLVa neurons (Fig. 5C). Figure $5 D$ shows data from 6 single units on which BMI was applied for at least $10 \mathrm{~min}$ and for which ITD curves were obtained with tone stimuli. Only 1 showed a clear increase in the half-peak width; the others showed little or no change. On average, the ratio of half-peak width to the stimulus period showed a slight, but not statistically significant, increase during BMI application $(56.1 \pm 7.1 \%$ without BMI, $60.8 \pm$ $11.1 \%$ with $\mathrm{BMI} ; n=6$ ). Both the trough : peak ratio and troughpeak difference were also unaffected by BMI application. The trough : peak ratio was $0.46 \pm 0.28$ before and $0.53 \pm 0.18$ after BMI was applied. The trough-peak difference was $17.9+11.1$ spikes/stimulus without BMI and $25.8 \pm 10.5$ spikes/stimulus with BMI.

\section{ICc core neurons}

As in the case of VLVa neurons, the administration of BMI increased responses of ICc core neurons both to favorable and to unfavorable ITDs, resulting in an upward shift of the entire ITD curve in non-normalized plots (see Figs. $4 A, 6,7 A$ ). These effects were consistently observed in all unit recordings we tested ( $n=24$; Table 1), with BFs ranging from 2.3 to $7.0 \mathrm{kHz}$. Effects of BMI on noise-evoked responses were similar to those on tone-evoked responses: both peaks and troughs were elevated (Fig. 6).

Although the effects of BMI on the firing rate of ICc corc neurons were somewhat similar to those on VLVa neurons (Fig. $7 A$ ), normalized plots show 2 differences: iontophoresis of BMI broadened the half-peak width and raised the trough : peak ratio in ICc core neurons (Fig. $7 B$ ). The ratio of half-peak width to the stimulus period increased in 11 of $12 \mathrm{ICc}$ core neurons we examined and slightly decreased in the remaining 1 neuron (Fig. $7 C$ ). The ratio was significantly larger during BMI application $(59.5 \pm 7.4 \%)$ than without BMI $(43.6 \pm 7.3 \% ; p<0.001)$.

Spike counts at troughs were nearly 0 in neurons shown in Figures 6 and 7 . When spike counts are 0 , the actual shape of ITD curves cannot be estimated, but are truncated at 0 in these neurons. One might therefore argue that BMI simply raises the entire ITD curve without changing its actual shape, and that once the trough is raised above 0 , the half-peak width appears to become broader. To test this hypothesis, we checked the cases where neurons showed spike responses to unfavorable ITDs. Five of the $12 \mathrm{ICc}$ core neurons showed spike responses to unfavorable ITDs (1.0-9.0 spikes/stimulus), and 4 of these 5 neurons showed broadening of peaks during BMI application. Although the remaining 7 neurons showed no or little spike responses (0.0-0.9 spikes/stimulus) to unfavorable ITDs, application of BMI raised the troughs above 0 in these neurons. We compared the half-peak width at the time when the troughs exceeded 0 with that at the time when the maximum effect of BMI was obtained. Five of the 7 neurons did increase the halfpeak width (e.g., see Fig. $4 A$ ), but the other 2 did not (see Fig. $9 B$, unit 713LG8).

Paired $t$ tests on each of the 12 units show that BMI application increased the trough: peak ratio, from $0.04 \pm 0.07$ to $0.40 \pm 0.13(n=12 ; p<0.001)$. In the presence of BMI, troughpeak difference increased in 6 neurons, decreased in 4 neurons, and remained unchanged in 2 neurons. On average, troughpeak difference was not significantly different before $(32.5 \pm$ 12.5 spikes/stimulus) and during BMI application (35.6 \pm 12.6 spikes/stimulus). Thus, the ITD curves of ICc core neurons became similar to those of NL and VLVa neurons in terms of
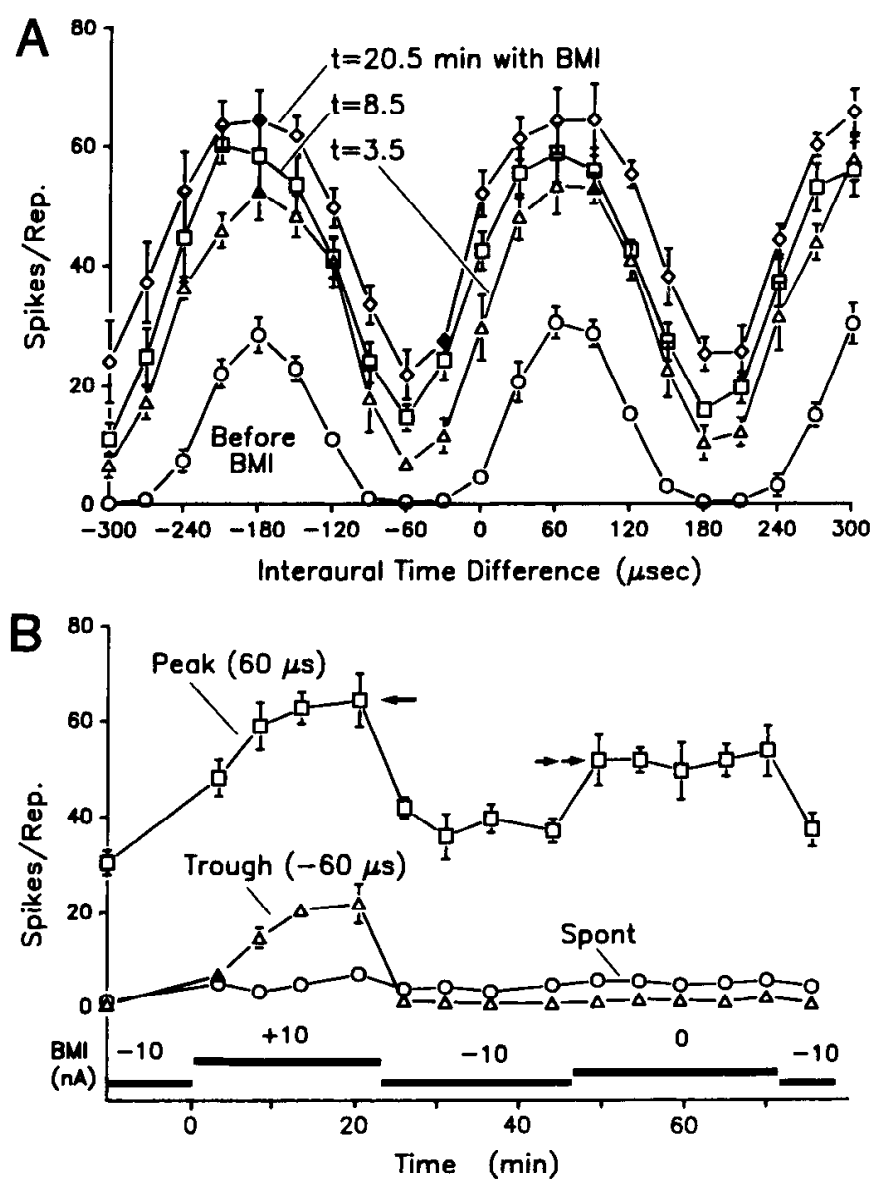

Figure 4. The time course of the effects of BMI on ITD selectivity of an isolated neuron in the right ICc core. $A$, The ITD curves obtained with tonal stimuli (neuron's BF, $4.1 \mathrm{kHz}$ ) before and during application of BMI with a $+10-n A$ ejecting current. Error bars indicate SD $(n=$ $5)$. $B$, The spike number at a favorable ITD $(60 \mu \mathrm{sec})$ and at an unfavorable $\operatorname{ITD}(-60 \mu \mathrm{sec})$ and spontaneous firing rate plotted against time. Horizontal bars (bottom) indicate the periods of current ejection: +10 , $+10 \mathrm{nA}$ ejecting current; $-10,-10 \mathrm{nA}$ retaining current; 0 , no current. Responses at the favorable ITD increased without any current (double arrow), presumably as a result of spontaneous leakage of BMI from the drug barrel. Note that this effect is smaller than that caused by a $+10-$ $\mathrm{nA}$ ejecting current (arrow).

half-peak width and trough : peak ratio (cf. ITD curves in Fig. $7 B$ with those in Figs. 2, 5).

\section{ICc lateral-shell neurons}

In ICc lateral-shell neurons, BMI application increased responses to tones at favorable ITDs and, to a lesser extent, at unfavorable ITDs (Fig. $8 A$ ). BMI application broadened the half-peak width in 11 of 13 cells tested (Fig. $8 B, C$ ). The ratio of half-peak width to the stimulus period was $42.6 \pm 4.0 \%$ before BMI application and increased to $53.2 \pm 6.3 \%$ when BMI was applied $(n=13 ; p<0.001)$. Five of the 13 cells had spike responses (1.8-7.8 spikes/stimulus) to unfavorable ITDs, and all 5 units broadened their peaks during BMI application (Fig. $9 A$, unit $713 \mathrm{~EB} 0$ in Fig. $9 B$ ). The other 8 neurons had no or little responses $(0.0-0.9$ spikes/stimulus) to unfavorable ITDs. In 6 of these 8 neurons, the half-peak width was larger at the time when the maximum effect of BMI was obtained than at the time when the trough became above 0 (e.g., Fig. $9 B$, unit 364CE0). 

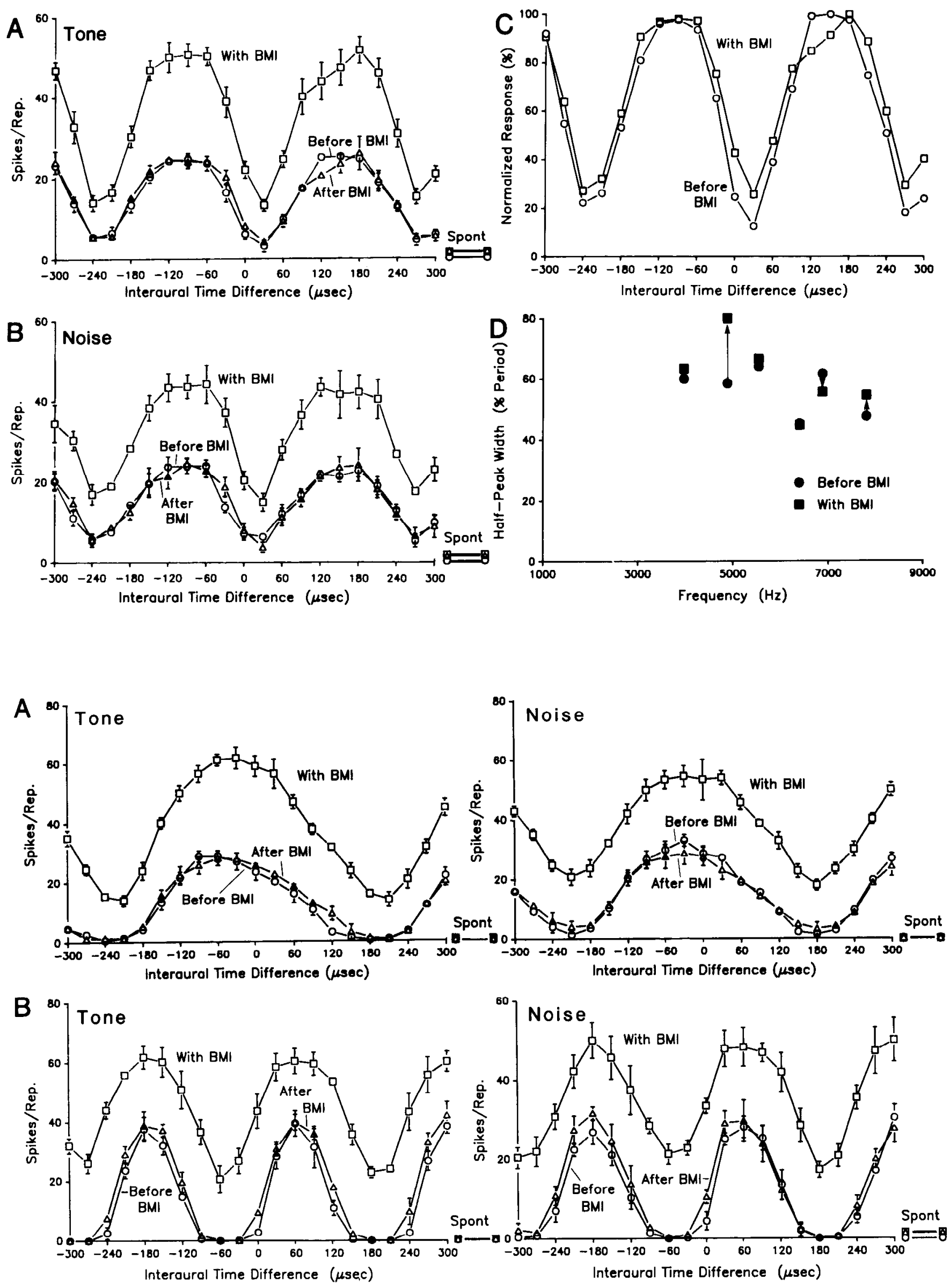

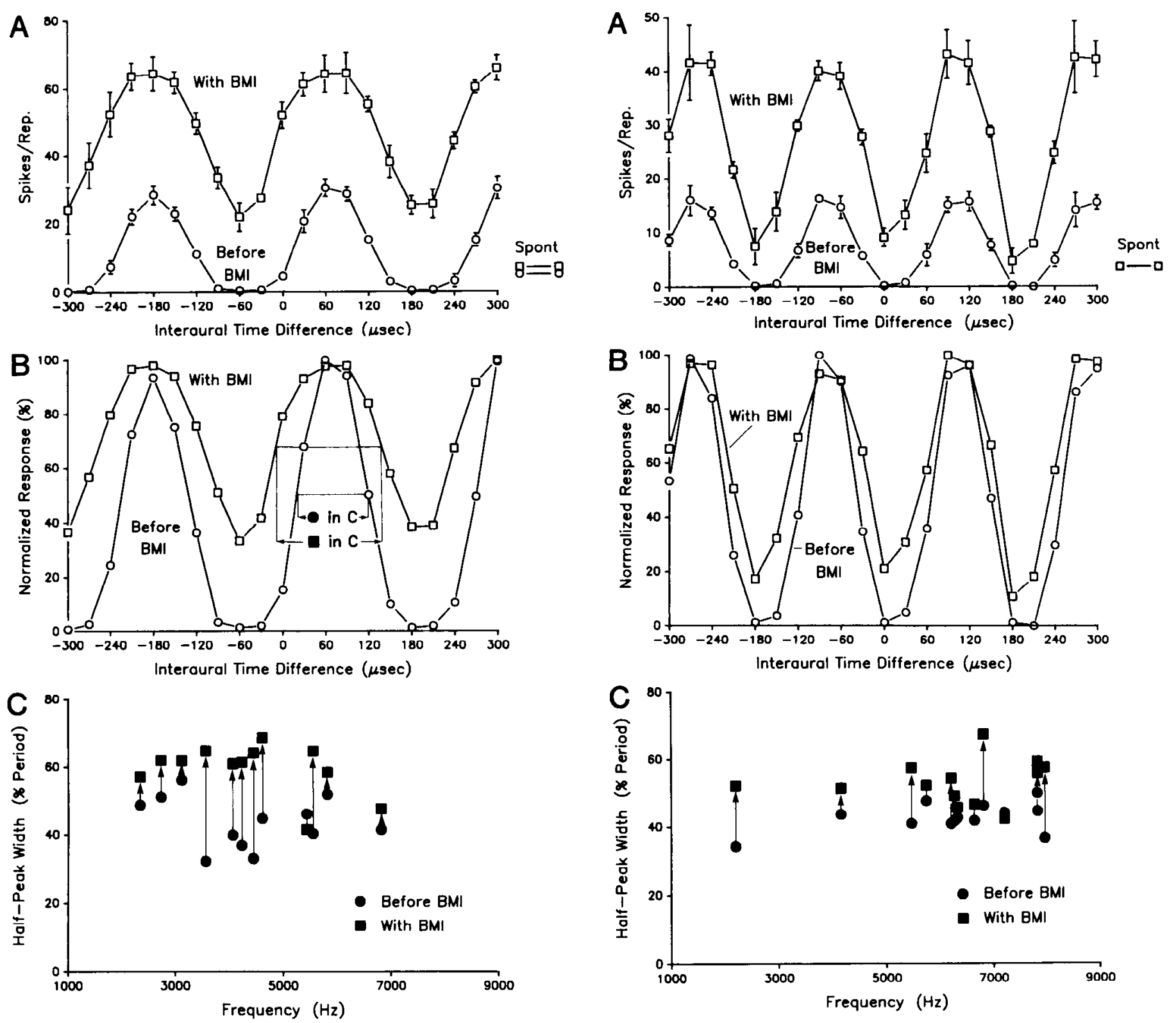

Figure 7. Effects of BMI on half-peak width of tonal ITD curves of ICc core neurons. $A$ and $B$, Non-normalized $(A)$ and normalized $(B)$ plots of ITD curves obtained from a neuron in the right ICc core. Tone bursts (neuron's BF, $4.1 \mathrm{kHz}$ ) were used as stimuli. Half-peak width was measured before and during BMI application $(B) . C$. The ratio of half-peak width to the period of the stimulus tone hefore and during BMI application. Data are collected from 12 isolated neurons to which a +10 -nA ejecting current for BMI was applied for at least $10 \mathrm{~min}$. Error bars indicate SD. Spont, spontaneous discharge rate.

Figure 8. Effects of BMI on half-peak width of tonal ITD curves of ICc lateral-shell neurons. $A$ and $B$, Non-normalized $(A)$ and normalized $(B)$ ITD curves of a neuron in the right ICc lateral shell. Tone bursts at the neuron's BF of $5.5 \mathrm{kHz}$ were used as stimuli. Spontaneous firing was constant before and during $3 \mathrm{MI}$ application. $C$, The ratio of halfpeak width to the period of the stimulus tone before and during BMI application. Data were obtained from 13 isolated neurons to which a + 10-nA ejecting current for BMI was applied for at least $10 \mathrm{~min}$. Error bars indicate SD. Spont, spontaneous discharge rate.

Figure 5. Effects of iontophoretically applied BMI on ITD selectivity of VLVa neurons. $A$ and $B$, Non-normalizcd ITD curves obtained with tone $(4.0 \mathrm{kHz}$, neuron's BF; $A)$ and noise $(B)$ in a left VLVa neuron. $B M I,+10 \mathrm{nA}$ for $10 \mathrm{~min}(A)$ and $12 \min (B)$. Recovery, $6 \mathrm{~min}(A)$ and 8 $\min (B)$ after currents for BMI stopped and switched to retaining currents of $-10 \mathrm{nA}$. Spont, spontaneous discharge rate. $C$, $A$ normalized version of the ITD curves in $A$ for the comparison of ITD curves before and during BMI injection. $D$, The ratio of half-peak width to the period of the stimulus tone before and during BMI application. Data are from 6 single units to which a +10 -nA ejecting current for BMI was applied for at least 10 min. Error bars indicate SD.

Figure 6. Effects of iontophoretic application of BMI on ITD selectivity of 2 neurons in the ICc core. The cell charted in $A$ was recorded in the left ICc core, and the cell charted in $B$. in the right ICc core. $A$ and $B$. Non-normalized ITD curves from 2 neurons before and during BMI application ( $+10 \mathrm{nA})$. Neuron's BF: $2.3 \mathrm{kHz}(A), 4.1 \mathrm{kHz}(B)$. Responses to BF tone and to noise of the same neurons are shown left and right. respectively. Error bars indicate SD. Spont, spontaneous discharge rate. 



Figure 9. Effects of BMI on ICc neurons with spike responses to unfavorable ITDs. $A$, Normalized ITD curves of a neuron in the right ICc lateral shell obtained with tonal stimuli (neuron's $\mathrm{BF}, 7.8 \mathrm{kHz}$ ) before and during $\mathrm{BMI}$ application $(+10 \mathrm{nA}, 6 \mathrm{~min})$. This neuron showed a response of 7.8 spikes/stimulus to unfavorable ITDs without BMI. BMI application broadened its half-peak width from 56.8 to $71.2 \mu \mathrm{sec} . B$, Examples of time course of change of half-peak width during BMI application. BMI application started at time 0 . Arrows indicate the time when troughs of ITD curves exceeded 0, i.e., when spike responses to the least-favorable ITDs began to appear. Unit $7 / 3 E B O$ from the lateral shell had spike responses even without BMI application. The other 2 units ( $713 L G 8$ in the core and $364 C E 0$ in the lateral shell) did not respond to unfavorable ITDs before BMI application and began to show spike responses $1 \mathrm{~min}$ after BMI was applied. The majority of $\mathrm{ICc}$ neurons (23 of 25) broadened their peaks after troughs exceeded 0 (e.g., units $713 E B O$ and $364 C E 0$ ), while the 2 cells did not broaden its peaks once the troughs were above 0 (e.g., unit $713 L G 8$ ).

The trough : peak ratio was raised by BMI: $0.08 \pm 0.08$ before BMI application compared to $0.30 \pm 0.23$ during BMI application $(p<0.001)$. BMI also increased trough-peak difference in all cells but 1 ( $20.4 \pm 7.4$ spikes/stimulus before vs. $34.7 \pm$ 16.6 spikes/stimulus during; $\rho<0.01$ ).

\section{ICX neurons}

In the ICx, iontophoresis of BMI increased tone-evoked responses to favorable ITDs, whereas responses to unfavorable ITDs remained unchanged or were elevated only slightly (Fig. $10 \mathrm{~A}$ ). On average, the ratio of half-peak width to the period of
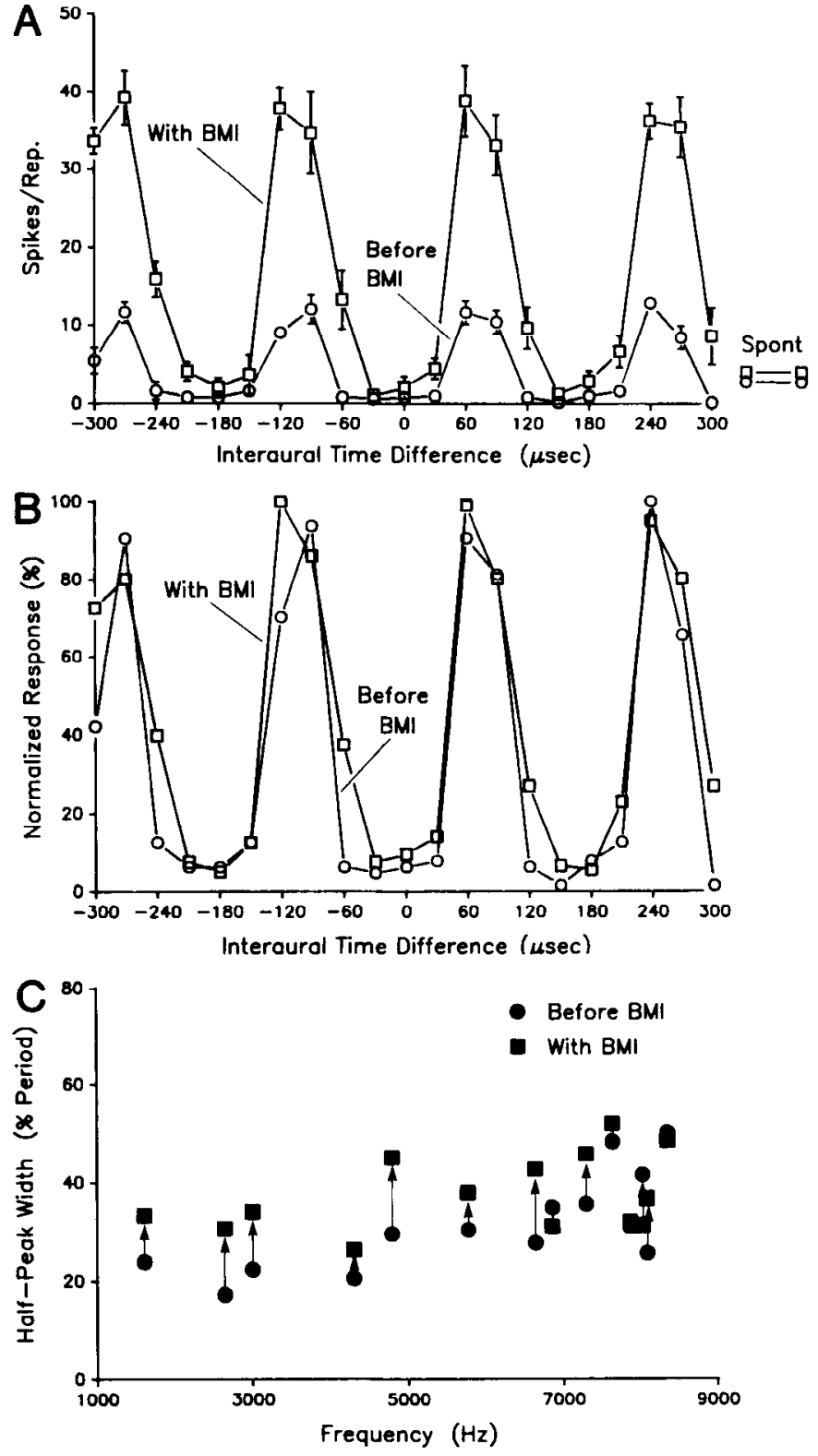

Figure 10. Effects of BMI on tone-evoked responses of ICx neurons. $A$, Non-normalized ITD curves of an ICx neuron obtained with tonal stimuli (neuron's BF, $5.8 \mathrm{kHz}$ ) before and during BMI application (+30 $\mathrm{nA}, 40 \mathrm{~min}$ ). Recordings were made in the right ICx. $B$, Normalized plots. $C$, The ratio of half-peak width to the period of the stimulus tone before and during BMI application. Data were collected from $14 \mathrm{ICx}$ neurons to which a +10 -nA ejecting current was applied for at least 10 min. Error bars indicate SD. Spont, spontaneous discharge rate.

the stimulus tone was $31.3 \pm 9.5 \%$ before BMI application and $37.7 \pm 7.6 \%$ during BMI application; this difference is statistically significant $(n=14 ; p<0.01)$. Peaks were broadened during BMI iontophoresis in 10 of 14 cells tested, and the other 4 cells did not change or decrease the half-peak width (Fig. $10 B, C)$. All of these 4 neurons had BFs above $6.8 \mathrm{kHz}$. Although the amount of disinhibition of troughs was small, there was an increase in the trough : peak ratio during the application of BMI $(0.03 \pm 0.05$ before vs. $0.09 \pm 0.10$ during; $p<0.001)$. The trough-peak difference increased in 11 units, decreased in 1 unit, and remained unchanged in 2 units. The average of trough-peak 
differences increased from $8.3( \pm 5.6)$ spikes/stimulus before BMI application to $23.9( \pm 13.6)$ spikes/stimulus during BMI application $(p<0.001)$.

In summary, BMI application altered ITD curves in all 4 nuclei examined, but the effects of BMI differed from nucleus to nucleus. Peaks and troughs were elevated in the VLVa and the ICc core. The half-peak width and trough : peak ratio, however, were affected only in the ICc core. The ICc lateral shell also showed disinhibition of both peaks and troughs in the prescnce of BMI, but peaks were disinhibited to a greater extent than troughs. This tendency is enhanced in the $\mathrm{ICx}$, where only peaks were elevated, and troughs remained almost unchanged. Lower-frequency ICx neurons consistently broadened their halfpeak width, whereas some high-frequency neurons did not.

\section{Relationship between monaural and binaural responses}

Monaural responses were compared with binaural responses before and during iontophoresis of BMI in 2 VLVa, 1 ICc core, and $2 \mathrm{ICc}$ lateral-shell neurons that had monaural responses. ICx neurons and some ICc lateral-shell neurons did not respond monaurally and were not examined for this analysis. Figure 11 shows an example of an ICc lateral-shell neuron. A larger spike number resulted from contralateral stimulation than from ipsilateral stimulation. Binaural responses to the most favorable ITD were greater than the sum of the responses to separate stimulation of the ipsilateral and contralateral ears (Fig. 11 A). As a measure of binaural interaction, calculated the summation ratio $(\mathrm{SR})$ :

$$
\mathrm{SR}=\frac{R_{b}-R_{s}}{\left(R_{i}-R_{s}\right)+\left(R_{c}-R_{s}\right)},
$$

where $R_{b}$ is the spike number of the binaural response to the most favorable ITD, $R_{i}$ and $R_{c}$ are responses evoked by separate stimulation of ipsilateral and contralateral ear, respectively, and $R_{\mathrm{v}}$ is the spontaneous firing rate. An SR value equal to 1 would mean summation between the inputs from the 2 ears; an SR value greater than 1, facilitation; and an SR value smaller than 1, occlusion (Goldberg and Brown, 1969). All cells except 1 VLVa neuron had an SR valuc grcater than 1 (range, 0.9-2.45; mean $\pm \mathrm{SD}, 1.58 \pm 0.56 ; n=5$ ). The $\mathrm{SR}$ value of the unit shown in Figure $11 \mathrm{~A}$ is 1.36 . In the 5 cells quantitatively examined, responses to unfavorable ITDs were smaller than both monaural response levels (Fig. $11 \mathrm{~A}$ ); this result was qualitatively confirmed in 8 additional cells ( 5 VLVa, 2 ICc core, and 1 ICc lateral-shell neurons). BMI application increased both binaural and monaural responses (Fig. $11 B$ ), with recovery observed after cessation of the ejecting current (Fig. 11C). The SR of the unit shown in Figure 11 decreased to 1.25 during BMI application. The SR value became smaller in all cells during BMI application (range, 0.65-1.25; mean $\pm \mathrm{SD}, 0.89 \pm 0.22 ; n=5$ ). The bottom of the trough remained below the spontaneous firing level in the presence of BMI (Fig. 11B). All neurons we tested from the VLVa, ICc core, and ICc lateral shell showed the same monaural and binaural relationship and showed similar responses to BMI application.

\section{Effects of BMl on phase ambiguity}

In the ICx, ITD curves obtained with noise stimuli were strikingly altered by BMI. BMI application elevated both the primary and the secondary peaks, as well as most troughs. The troughs adjacent to the primary peak often remained relatively low (Fig.
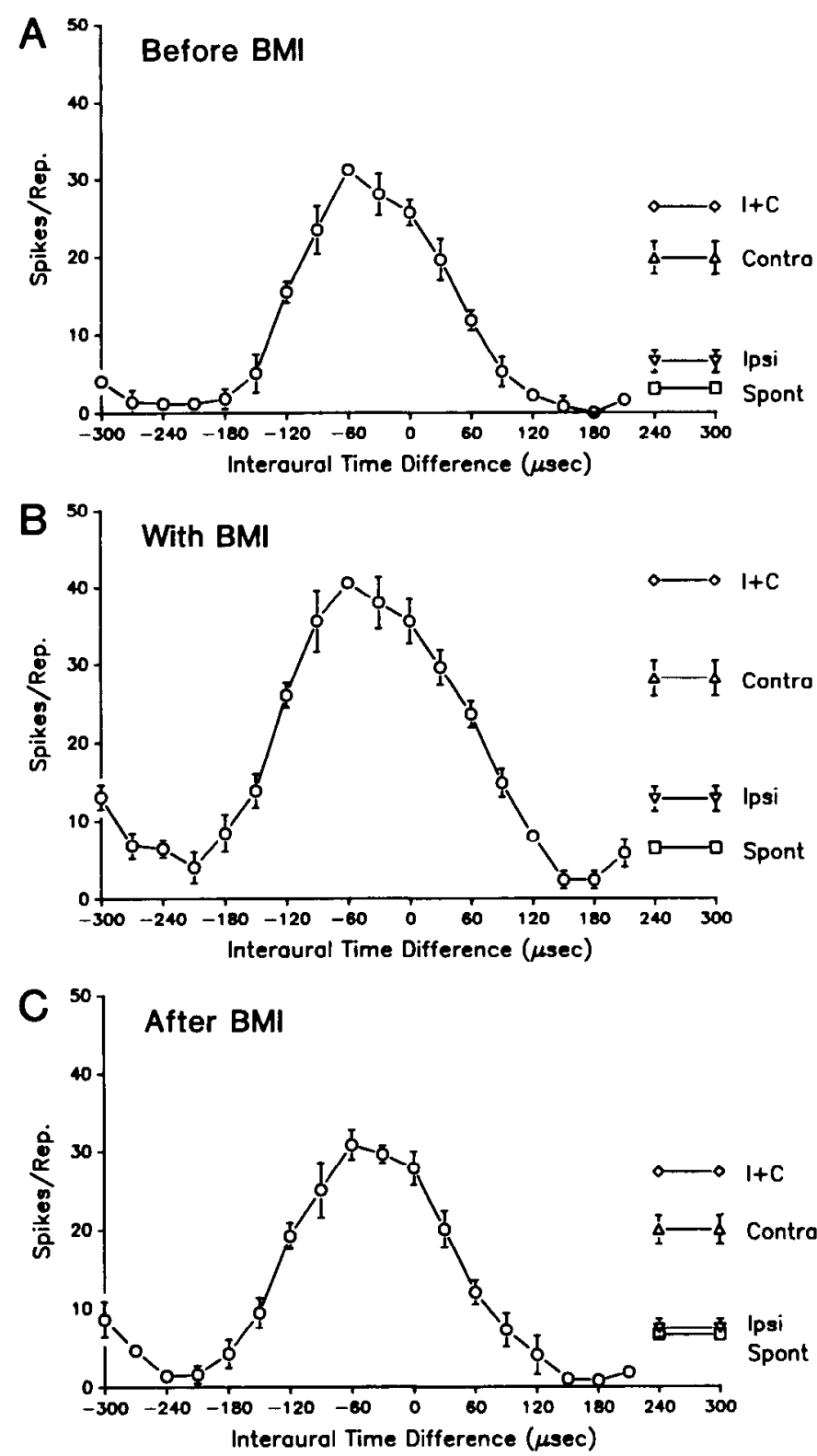

Figure 11. The comparison of monaural with binaural responses before and during BMI application. An example of a neuron isolated from the right ICc lateral shell is shown here. Noise bursts were used as stimuli. Bars on the right show the firing levels of spontaneous activity (Spont), responses to ipsilateral (Ipsi), and contralateral (Contra) stimulation and the algebraic sum of ipsilateral and contralateral responses $(I+C)$. $A$, Before BMI application. $B$, During BMI application at +30 $\mathrm{nA}$ for $4 \mathrm{~min}$. $C$, Recovery data obtained 4 min after cessation of BMI current application. Error bars indicate SD.

$12 A, B)$. In the ICx neuron shown in Figure $12 A-C$, the height of the secondary peaks was about $20 \%$ of that of the primary peak before BMI application. All 4 secondary peaks gradually increased over $20 \mathrm{~min}$ of BMI application (Fig. 12C). One of the secondary peaks (at $+120 \mu \mathrm{sec}$ of ITD) reached $80 \%$ of the primary peak. Figure $12 \mathrm{D}$ shows data from 56 secondary peaks in 17 ICx neurons. Before BMI application, 32 of 56 secondary peaks were smaller than $50 \%$ of the primary peaks, while only 4 peaks remained smaller than 50\% during BMI application. The ratio of heights of secondary peaks to the primary peak was increased from $50.3 \pm 19.9 \%$ without BMI to $73.2 \pm 13.1 \%$ 



Figure 12. Effects of BMI on secondary-peak suppression. $A$ and $B$, Non-normalized $(A)$ and normalized $(B)$ ITD curves of a neuron in the right ICx before and during BMI application $(+10 \mathrm{nA}, 17 \mathrm{~min})$. Noise bursts were used as stimuli. The arrow in $A$ shows the spontaneous firing rate, which remained constant before and during BMI application. $C$, Time course of effects of BMI on the secondary peak suppression of the cell shown in $A$ and $B$. The ratio of each of 4 secondary peaks to the primary peak was plotted against time. $D$, The ratio of heights of the sccondary peaks to the primary peak before and during BMI application. Data are from 56 secondary peaks in 17 ICx neurons and are plotted as bins of $10 \%$ width. Error bars indicate SD.

with BMI $(n=56 ; p<0.001$, paired $t$ test after arcsine transformation).

\section{Effects of BMl on the temporal discharge pattern}

The temporal discharge pattern of auditory responses to tone bursts at the most favorable ITDs was categorized as phasic (on-transient response only), phasic-tonic (on response followed by sustained response or sustained response with adaptation), or tonic (sustained response without prior phasic component; Table 2). As long as neurons were stimulated with BF and the most favorable ITD, we never observed other types of responses, such as tonic inhibition. The response characteristic was determined by visual inspection from a peristimulus time histogram, displayed in a 3-D manner (Figs. 13, 14). Before the administration of BMI, the majority ( $83 \%$ ) of VLVa and ICc core neurons showed tonic responses (see Figs. 13A, 16), while the remaining cells were of the phasic-tonic type. In the ICc lateral shell, phasic-tonic-type responses predominated (77\%; Fig. 13B). No cells in the VLVa, ICc core, or ICc lateral shell showed phasic responses. In the ICx, however, more than half (57\%) of the neurons showed phasic responses (Fig. 14, top), and $29 \%$ of the neurons showed phasic-tonic response. Thus, the majority of ICx neurons $(86 \%)$ had phasic components in their responses, and only $14 \%$ showed tonic responses.

The administration of BMI changed this response pattern (Table 2). Many of the phasic-tonic-type neurons became tonic

Table 2. Effects of BMI on the temporal characteristics of responses to tones

\begin{tabular}{|c|c|c|c|c|c|c|}
\hline & \multicolumn{3}{|c|}{ Before BMI } & \multicolumn{3}{|c|}{ During BMI } \\
\hline & Phasic & $\begin{array}{l}\text { Phasic- } \\
\text { tonic }\end{array}$ & Tonic & Phasic & $\begin{array}{l}\text { Phasic- } \\
\text { tonic }\end{array}$ & Tonic \\
\hline $\operatorname{VLVa}(n=6)$ & 0 & 17 & 83 & 0 & 17 & 83 \\
\hline ICc core $(n=12)$ & 0 & 17 & 83 & 0 & 0 & 100 \\
\hline ICc lateral shell $(n=13)$ & 0 & 77 & 23 & 0 & 8 & 92 \\
\hline $\operatorname{ICx}(n=14)$ & 57 & 29 & 14 & 0 & 79 & 21 \\
\hline
\end{tabular}

This table shows the percentage of cells observed. The number of cells analyzed is shown in parentheses on the left. All data are from single units, to which a +10-nA ejecting current for BMI was applied for at least $10 \mathrm{~min}$. 
A

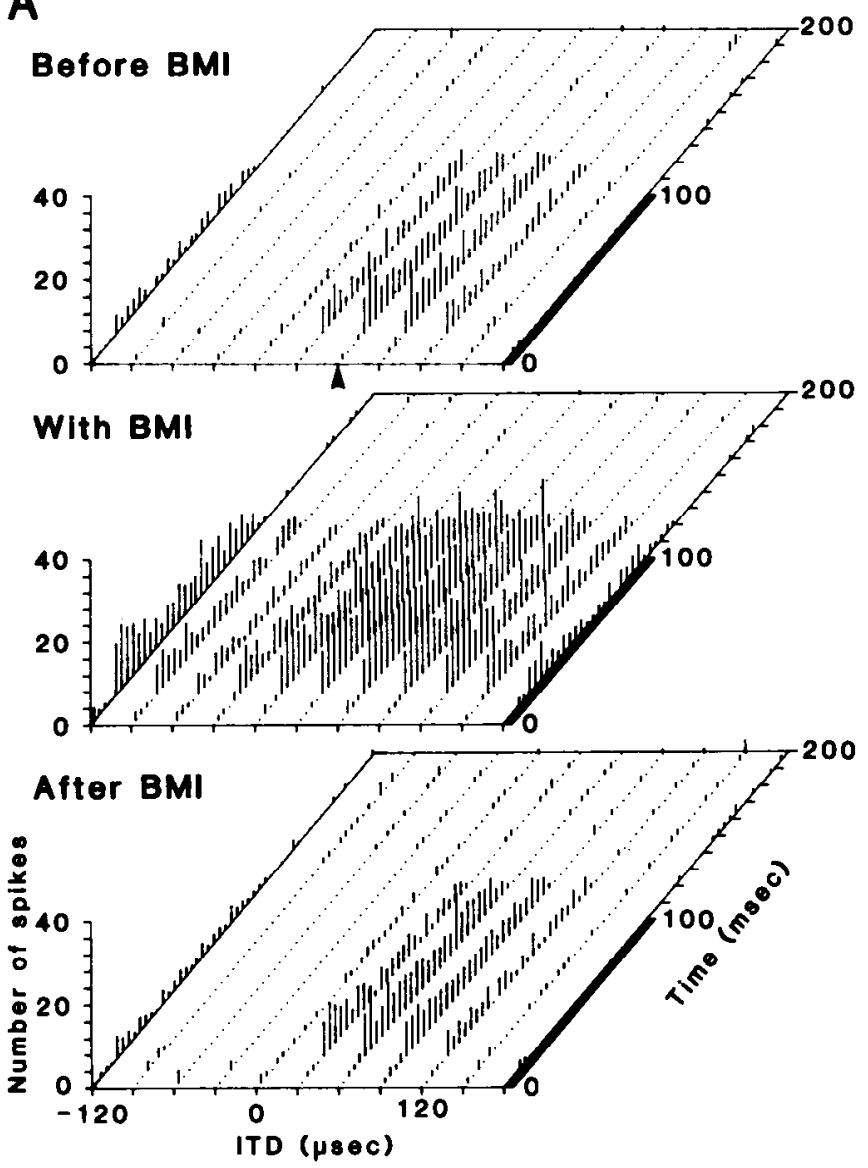

B

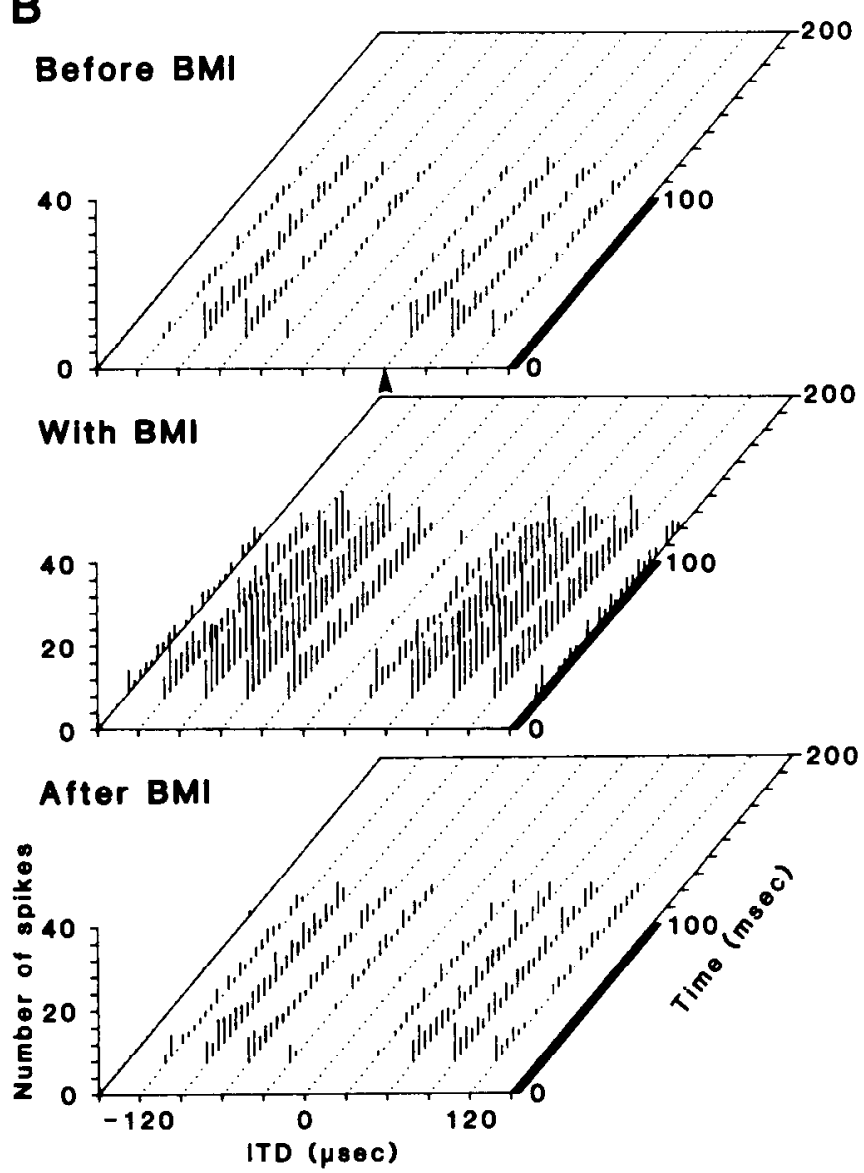

Figure 13. Peristimulus time histograms of an ICc core neuron $(A)$ and a läteral-shell neuron $(B)$ from right hemisphere. Tone bursts at neuron's BF $(A, 4.1 \mathrm{kHz} ; B, 6.2 \mathrm{kHz})$ were used as stimuli. Data are shown as 3-D plots of neural responses before, during, and after administration of BMI. $x$-axis, ITD ( $\mu \mathrm{sec}) ; y$-axis, the number of spike in 4-msec bins; $z$-axis, time (msec after the onset of auditory stimuli). Solid bars on the right side indicate the period of auditory stimuli (0-100 msec). Before BMI application, temporal discharge patterns at the most favorable ITDs (arrowheads) were tonic $(A)$ and phasic-tonic $(B)$. Both neurons showed tonic responses in the presence of BMI $(8.5$ min for cell $A, 12$ min for cell $B)$. ITD curves for the cell $A$ are shown in Figure $4 A$.

(Fig. 13B), and all phasic units in the ICx became either phasictonic (Fig. 13) or tonic.

\section{Effects of GABA}

In all recordings from the VLVa and the inferior colliculus (15 single and 5 multiple units; Table 1), iontophoretic application of GABA with a +5 to $+40-n A$ ejecting current suppressed spontaneous discharges and auditory responses (Figs. 15-17). In the cells shown in Figures 15-17, spontaneous discharge rates were very low, but not 0 , before GABA application, and the administration of GABA totally silenced these cells. In the VLVa neuron shown in Figure 15, +20-nA GABA suppressed responses only at unfavorable ITDs, and + 40-nA GABA suppressed responses to both unfavorable and favorable ITDs (Fig. $15 B)$. In some cases, both spontaneous activity and auditory responses were inhibited simply by switching off the retaining current. As in the case of BMI, this effect was likely to be caused by leakage of the drug from the pipette. The effects of GABA were fully reversible (Figs. 15-17). The latency to maximum effect of GABA and its recovery were faster than those of BMI. This is presumably because BMI must compete with naturally released GABA, and because BMI has a larger molecular weight than GABA and hence a slower diffusion rate. In VLVa neurons, suppression of auditory responses (Fig. 15A) narrowed the halfpeak width (Fig. 15C). The resulting ITD curve is very similar to that of ICc neurons.

When we applied GABA to neurons with a tonic discharge pattern, responses in the first $10-20 \mathrm{msec}$ tended to be more resistant to GABA than the later responses. The temporal discharge pattern thus became phasic or phasic-tonic. An example of an ICc core neuron is shown in Figure 16.

In all neurons tested ( $n=10)$, the effects of GABA and those of BMI antagonized each other. Figure 17 shows an example of an ICc core neuron. Without any drugs applied ("before" in $A$, arrow 1 in $B$ ), this cell showed a response of 27.4 spikes/stimulus (mean, 5 stimulus repetitions) to a favorable ITD of $-60 \mu \mathrm{sec}$. Iontophoresis of GABA with a +10-nA current strongly suppressed this response within 8 min to 2.6 spikes/stimulus ("with GABA" in $A$, arrow 2 in $B$ ). By contrast, BMI at $+10 \mathrm{nA}$ increased the response by $220 \%$ to 61.0 spikes/stimulus ("with BMI" in $A$, arrow 3 in $B$ ). When BMI and GABA were applied simultaneously, auditory responses remained constant even 12 min after the currents started ("with BMI + GABA" in $A$, arrow 4 in $B$ ). The effect of BMI on the suppression of auditory responses by GABA was also reversible; when we stopped current for BMI, the suppressive effects of GABA reappeared (arrow 5 in $B$ ). 

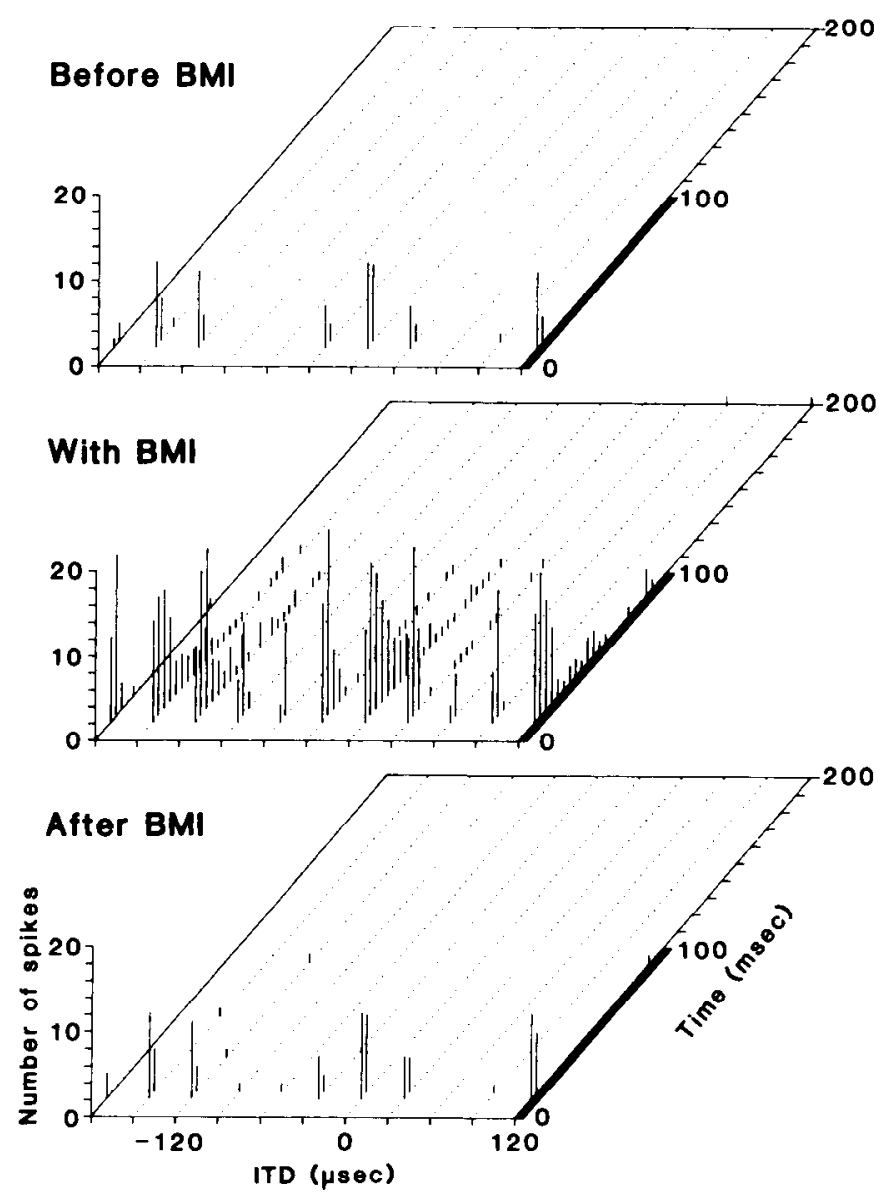

Figure 14. Peristimulus time histograms of responses of a neuron in the right ICx to tone bursts (neuron's BF, $7.3 \mathrm{kHz}$ ) before, during, and after BMI application. Data were obtained $5 \mathrm{~min}$ after the start of a $+20-n A$ ejecting current of BMI. After 20 min of ejection, current was switched from $+20 \mathrm{nA}$ to $-10 \mathrm{nA}$ retaining current. Recovery data were obtained $14 \mathrm{~min}$ after retaining current started. This neuron showed phasic responses without BMI, while tonic components appeared during BMI application. See Figure 13 for conventions.

\section{Discussion}

\section{Technical consideration}

One of the pitfalls of microiontophoresis is the occurrence of artifacts of various origins such as current and $\mathrm{pH}$ (Bloom, 1974; Stone, 1985). In our preliminary experiments, we sometimes observed obvious current effects when we used higher current intensities $(+75$ to $+100 \mathrm{nA})$. These effects appeared or disappeared upon start or cessation of an ejecting current, and the direction of the effects (suppression or activation) often reversed when the polarity of the current was reversed. The effects reported in this paper, however, were not produced by current itself, because (1) the effects grew gradually, and it took up to $30 \mathrm{~min}$ to reach the maximum or to recover the original level (Figs. 4, 12, 15, 17); (2) the threshold current was raised, and the time to reach the maximum effect became longer when we used higher retaining currents prior to BMI application (data not shown); (3) effects similar to iontophoresis were occasionally produced by switching off retaining currents, presumably as a result of spontaneous leakage of drugs (Fig. 4B); and (4) current ejection from the vehicle-containing pipettes did not affect the responses. The last finding also excludes the possibility that the


Figure 15. Effects of GABA on ITD selectivity of a neuron in the left VLVa. Tone bursts of neuron's BF $(4.0 \mathrm{kHz})$ were used. Effects of BMI on this cell are shown in Figure $5 A-C . A$. Non-normalized ITD curves obtained before and during GABA application $(+10 \mathrm{nA}, 5 \mathrm{~min}) . B$, The number of spikes at a peak (ITD, $-90 \mu \mathrm{sec}$ ) and a trough (ITD, 30 $\mu \mathrm{sec}$ ) and spontaneous discharges (Spont) are plotted against time. Solid horizontal bars indicate the period of drug application. $C$, Normalized plots of the ITD curves shown in A. Error bars indicate SD.

observed effects were caused by low $\mathrm{pH}\left(\mathrm{H}^{+}\right)$or other ions in the solvent (e.g., $\mathrm{Na}^{+}$).

Bicuculline is now widely accepted as a selective antagonist of GABA. It competes with GABA for the $\mathrm{GABA}_{\mathrm{A}}$ receptor site of the GABA receptor/benzodiazepine receptor/chloride channel complex and reduces GABA-mediated inhibition in postsynaptic cells (Andrews and Johnston, 1979; Bormann, 1988). A quaternary derivative, BMI, is more potent, more water soluble (Johnston et al., 1972; Pong and Graham, 1972), and more stable (Olsen et al., 1975) than bicuculline. The following 2 observations indicate that the effects reported in this paper were caused by reduction of GABA-mediated inhibition rather than 

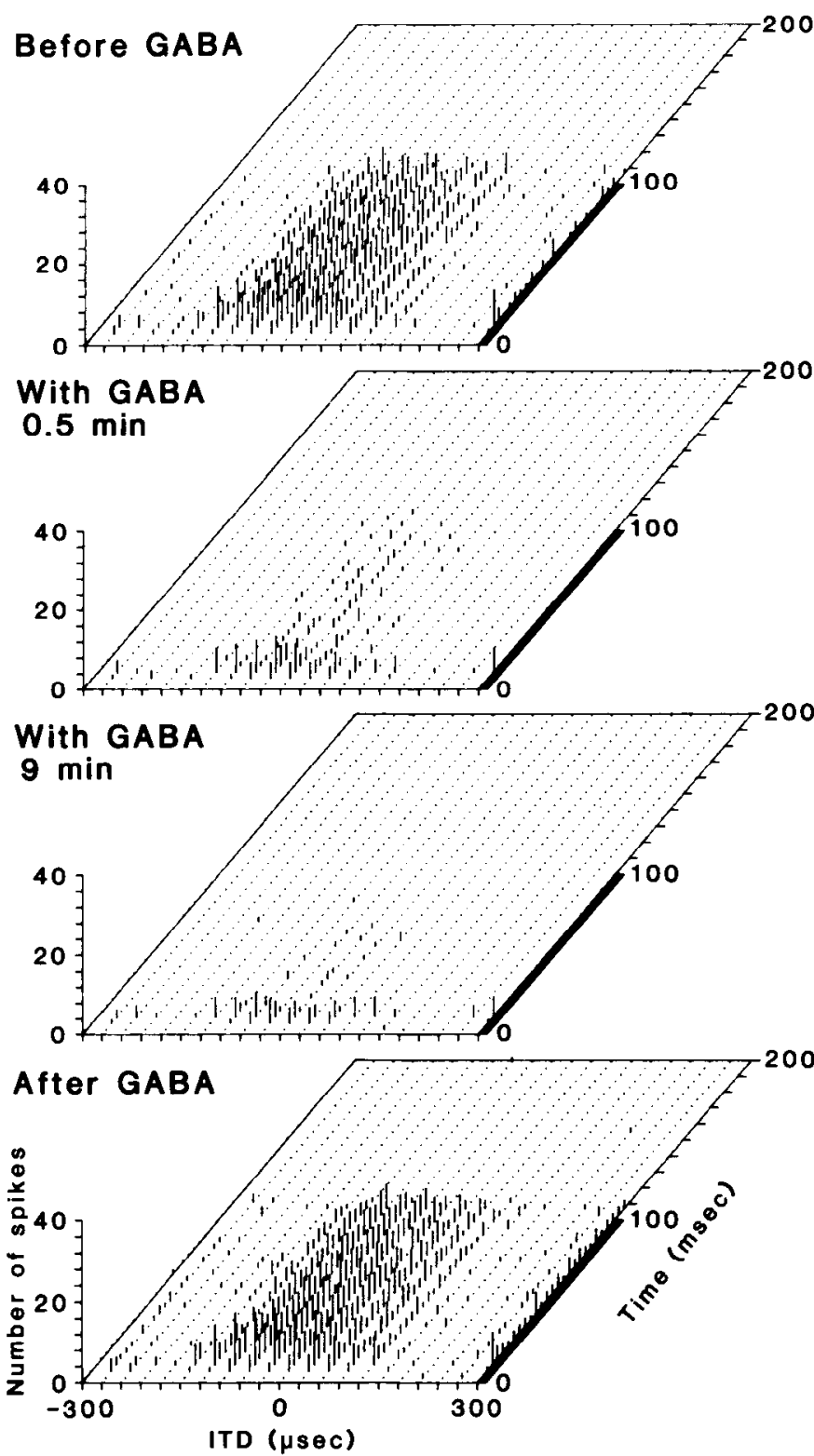

Figure 16. Peristimulus time histograms of responses of a neuron isolated from the left ICc core before, during, and after GABA application. Tone bursts (neuron's BF, $2.3 \mathrm{kHz}$ ) were used as stimuli. ITD curves of this neuron arc shown in Figure 17. Ejecting current for GABA, +10 $\mathrm{nA}$. Recovery data were obtained $1 \mathrm{~min}$ after current for GABA was stopped. See Figure 13 for conventions.

dircct cxcitatory effects, if any, of BMI or its degradation products on the neurons. First, iontophoretically applied BMI increased auditory responses 2-10 times, with no or little effect on spontaneous firing. Even in cells whose spontaneous discharge was elevated, the increase could not account for the degree and nature of changes in auditory responses (e.g., separate effects on peaks and troughs in ICx neurons). Second, BMI blocked the depressant effect of iontophoretically applied GABA (Fig. 17).

All neurons tested were affected by BMI and GABA. It is possible that our electrodes may have selected only particular populations of neurons. It is unlikely, however, that we recorded only from non-GABAergic cells and not from GABAergic inhibitory neurons, because the range of cell sizes of these 2 groups
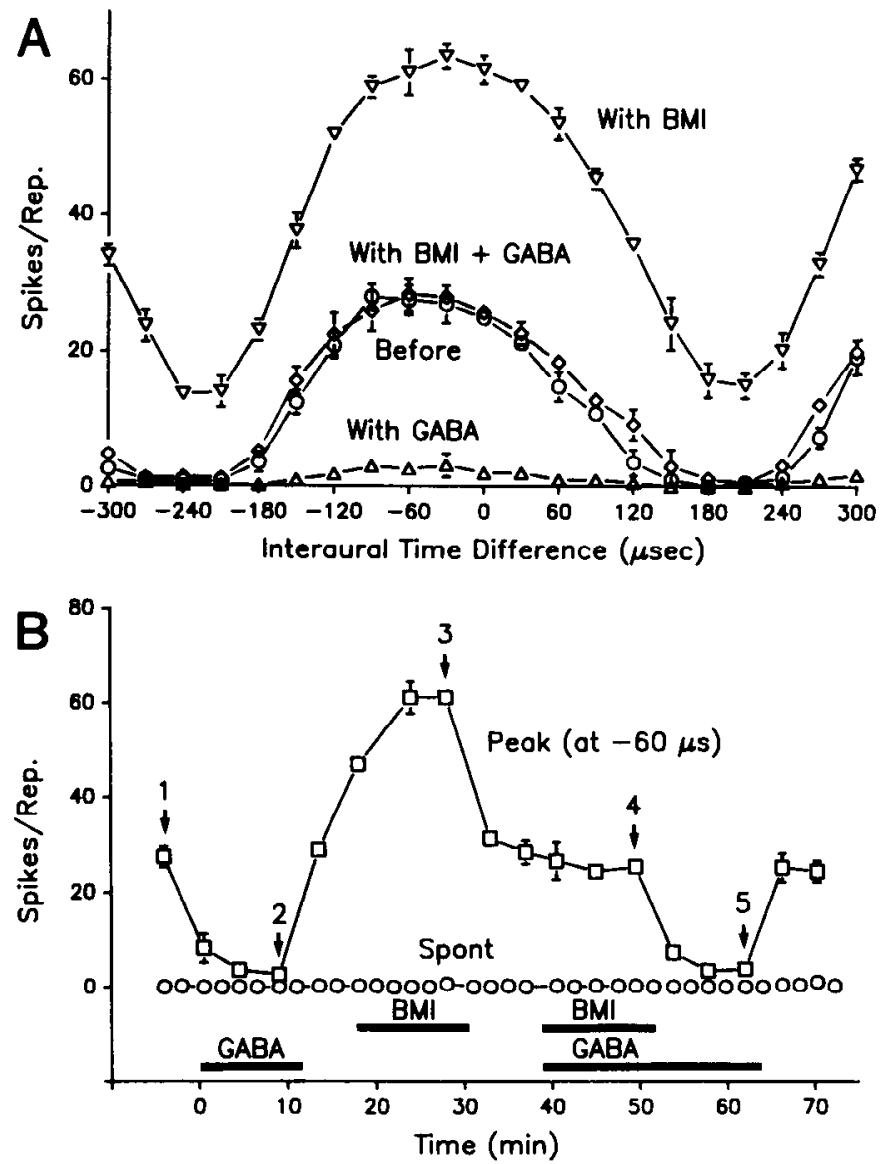

Figure 17. Antagonism between BMI and GABA examined in a neuron recorded from the left ICc core. Tone bursts (neuron's BF, $2.3 \mathrm{kHz}$ ) were used as stimuli. $A$, Non-normalized ITD curves. $B$, The number of spikes at a peak (ITD, $-60 \mu \mathrm{sec}$ ) and spontaneous discharges (Spont) of the cell are plotted against time. When administered separately, GABA decreased (arrow 2) and BMI increased (arrow 3) auditory responses. When both drugs were applied simultaneously, the responses remained unchanged even after $12 \mathrm{~min}$ of ejection (arrow 4). Fjecting current for GABA and BMI was $+10 \mathrm{nA}$. Horizontal bars indicate the periods of drug application. Error bars indicate SD.

of neurons completely overlaps in the inferior colliculus and the VLVa of the barn owl (Carr et al., 1989). It is not surprising that all neurons are affected by BMI and GABA, because both GABAergic and non-GABAergic neurons in the inferior colliculus and the VLVa appear to be innervated by GABAergic terminals (Carr et al., 1989).

\section{Source of inhibitory inputs}

BMI disinhibits auditory responses without changing spontaneous firing in most neurons. This indicates that most, if not all, GABAergic neurons receive auditory inputs, and that GABA is not released tonically, but rather only when these neurons are activated by auditory stimuli. Although the VLVa and all subnuclei of the inferior colliculus contain GABAergic neurons (Carr et al., 1989), it remains to be determined whether the inhibition that we blocked with BMI is mediated by these local neurons in each nucleus or by projection neurons from lower or higher nuclei.

Inhibition by descending projections, however, does not appear to occur in the ITD pathway. Despite recent extensive hodological analyses (Takahashi and Konishi, 1988a,b; Takahashi et al., 1989), no convincing indication of descending pro- 
jections has been obtained in the auditory brainstem of the barn owl, except projections from the superior olive to the nucleus magnocellularis (NM; Carr et al., 1989) and from the nucleus lemnisci lateralis, pars ventralis (LLv) to the superior olive (T. Takahashi, personal communication). Although the possibility of a forebrain projection to the inferior colliculus has not been examined in the barn owl, it has been reported that, in the canary, the primary forebrain auditory area field L does not project to the inferior colliculus (Kelly and Nottebohm, 1979).

\section{Contributions of GABA-mediated inhibition to ITD selectivity}

The quasi-sinusoidal shape of ITD curves derives initially from the timing of phase-locked spikes in the NM and from the responses of NL neurons as coincidence detectors (Konishi, 1986; Sullivan and Konishi, 1986; Carr and Konishi, 1988). These mechanisms alone can produce the cyclic form of ITD curves. Colburn et al. (1990) have recently proposed a model to explain the ITD curves of the dog medial superior olivary neurons that were obtained by Goldberg and Brown (1969). This model simulates the responses of the neurons to ITDs without any inhibitory component. In contrast, Grün et al. (1990) incorporate inhibition to simulate the binaural interaction in the owl's NL. Their model predicts that blockade of inhibition reduces ITD selectivity in the NL. The techniques used in the present study, however, cannot easily be applied to the NL in order to test this prediction, because extracellular recording from NL cells is extremely difficult. In our preliminary experiments, we instead injected BMI $(0.4-0.7 \mu \mathrm{l})$ by pressure into the NL, while recording from neurons in the ICc core contralateral to the injected NL. Injection of BMI, but not saline, into the NL led to a flattening of ITD curves in many ICc core cells. This effect was frequency specific: only ICc core neurons tuned to a frequency similar to that at the NL injection site were affected (I. Fujita and M. Konishi, unpublished observations). This result, together with immunocytochemical demonstration of GABAergic terminals around NL cell bodies (Carr et al., 1989), suggests that GABAergic inhibition affects ITD selectivity of NL cells. It is yet to be determined how GABAergic inhibition actually contributes to the generation of ITD selectivity in the NL (see also below).

A comparison of half-peak width in different nuclei indicates that neurons are more sharply tuned to ITD in higher-order nuclei, though an improvement of ITD tuning does not occur in every transition from lower to higher stations. Sharpening occurs only in the ICc core and the ICx and appears to involve GABAergic inhibition because BMI broadened the peak width. BMI application also broadened the half-peak width in the ICc lateral shell (Fig. 8). Because no additional sharpening of ITD tuning occurs between the core and the shell, this finding suggests that the ICc core may not be the sole source of inputs to the lateral shell.

The ratio of half-peak width to the period of the stimulus tone does not change with frequency in the ICc core, but for ICX neurons, this ratio is smaller in the lower-frequency range than in the high-frequency range (Fig. 3B). Together with the observation that some ICx neurons with higher BFs did not increase their half-peak width in the presence of BMI (Fig. 10C), these results suggest that the ITD tuning of lower-frequency neurons is sharpened by inhibition within the ICx, while the ITD selectivity of neurons with higher BFs is not sharpened or is at least less improved by inhibition beyond the ICc.
The trough: peak ratio and trough-peak difference are the other aspects of ITD curves we measured. The trough-peak difference is larger and the trough : peak ratio smaller in the $\mathrm{ICc}$ core than in the VLVa. As we ascend from the ICc core to the ICc lateral shell to the ICx, the difference decreases. BMI application raises both peaks and troughs in the VLVa, the ICC core, and the ICc lateral shell. In the ICx, only peaks are elevated with BMI, but this is presumably because the troughs are already inhibited in the ICc and cannot be disinhibited (see discussion in the next section). Thus, within the ITD pathway, GABAcrgic inhibition appears to operate both at favorable and at unfavorable ITDs. We suggest that this global inhibition could be important for the encoding of ITDs in the following manner: Neurons tuned to different ITDs are arranged systematically so as to form a map of ITDs in the ICC and the ICx. A sound stimulus of a given ITD activates a population of neurons whose distribution in the map presumably signals ITDs to the next station. If inhibition reduces the discharge of suboptimally activated neurons to insignificant levels, the locus of optimally stimulated neurons would stand out in the map. In other words, inhibition reduces "noise" in the distribution of excitation.

Inhibition operating simultaneously with excitation under optimal stimulus conditions is found in many aspects of central sensory processing, for example, spatial receptive field of retinal ganglion cells (Rodieck, 1965), orientation selectivity of visual cortex neurons (Ferster, 1986), somatosensory cortical receptive field (Gardner and Constanzos, 1980), and IID selectivity of the barn owl auditory brainstem and the inferior colliculus (I. Fujita and $\mathbf{M}$. Konishi, unpublished observations). This type of inhibition can enhance spatial contrast (see above; Rodieck, 1965) and improve temporal resolution (see below). It could also make other contributions that may differ from case to case; for example, in the $\mathrm{ICx}$, it contributes to the elimination of phase ambiguity, as discussed below.

\section{Within-ITD and cross-ITD inhibition}

In this section, we shall focus our discussions on the results obtained with tone stimuli. As pointed out previously, auditory stimuli drive inhibitory neurons. These inhibitory neurons may not be tuned to ITD and act independently of ITD. Here, we call this type of inhibition "general" or "ITD-nonspecific" inhibition. Alternatively, they might also be ITD selective, and inhibition would operate either between neurons tuned to the same frequency and ITDs ("within-ITD inhibition") or act laterally on neurons tuned to the same frequency but to different ITDs ("cross-ITD inhibition").

VLVa and ICc neurons increased their responses both to favorable and to unfavorable ITDs during BMI application. This result can be explained by assuming general (ITD-nonspecific) inhibition mediated by GABA; some observations, however, do not support this. First, the time course and the degree of BMI effects differ between the peak and trough (Fig. 4A), and at least in some ICc core neurons, low doses of BMI or GABA can affect either trough or peak alone, leaving the other unaffected (Figs. $4 B, 15 B$ ). These results suggest that inhibitory synapses responsible for peaks and troughs are not identical and are differently distributed on the soma and dendrites. Second, no ITD-insensitive neurons were found in the VLVa, ICc core, ICc lateral shell, and ICx, yet such neurons would be required if there were general inhibition. Another possible, more likely mechanism is a combination of cross-ITD inhibition and within-ITD inhibition. This mechanism requires ITD-selective in- 
hibitory neurons and different inhibitory synapses for peak and trough.

The failure of BMI to disinhibit the troughs in ICx neurons is most likely due to the lack of excitatory inputs from ICc neurons at unfavorable ITDs, though within-ITD inhibition could also explain the results. In the within-ITD inhibition scheme, principal and inhibitory neurons are maximally activated by the same ITD. Therefore, principal neurons receive both strong excitatory and inhibitory inputs at favorable ITDs. At unfavorable ITDs when excitatory inputs are small, the ncurons receive only minimal inhibition. If we remove inhibition by the application of BMI, responses would be greatly enhanced at favorable ITDs but not at unfavorable ITDs. A cross-correlation analysis of 2 simultaneously recorded units would help to answer the question of cross- versus within-ITD inhibition. Intracellular staining of neurons combined with immunocytochemistry would also shed light on this issue by revealing how far the axons of the GABAergic interneurons extend and whether this projection remains within an iso-ITD column or spans several iso-ITD columns.

\section{Suppression of response to unfavorable ITDS}

Troughs of ITD curves were below the monaural response level in the VLVa and ICc. BMI elevated both the monaural responses and the troughs, but the latter never exceeded the former (Fig. 11). These results suggest that the reduction in firing is caused by a mechanism other than $\mathrm{GABA}_{\mathrm{A}}$ receptor-mediated inhibition in these nuclei, or that it occurs downstream in the pathway (i.e., in the NL). A similar relationship between monaural and binaural responses to unfavorable ITDs has also been described at several stages of the mammalian auditory system, including the medial superior olive (Goldberg and Brown, 1969), the dorsal nucleus of the lateral lemniscus (Brugge et al., 1970), and the inferior colliculus (Rose et al., 1966; Kuwada et al., 1979). Although these authors suggest that "inhibitory processes" operate to produce this phenomenon, the reduction of firing rate below the monaural response level can be achieved by noninhibitory processes. It is an open question whether inhibition is involved in this suppression of discharges below the monaural response level.

\section{Elimination of phase ambiguity}

When stimulated with broad-band noise or 2-tone stimuli, ICx neurons resolve phase ambiguity by suppressing the peaks at phase-equivalent ITDs and/or facilitating the peak at the true ITD (Takahashi and Konishi, 1986). Disinhibition of the secondary peaks by the administration of BMI (Fig. 12) clearly demonstrates that this phenomenon has a central origin and is not due to 2-tone suppression at the periphery. A mechanism for the elimination of phase ambiguity has been proposed as follows by Takahashi and Konishi (1986): An ICx neuron receives converging inputs from an array of ICc neurons with different BFs (Wagner et al., 1987). These ICc neurons have 1 common, array-specific ITD, but the periodicity of cyclic change differs among them. Therefore, the postsynaptic ICx neurons sum excitatory inputs at the array-specific ITD. At other ITDs, excitatory inputs from a frequency channel may be canceled out by inhibitory inputs from other frequency channels. These authors assume that ITD curves are composed of "excitatory" peaks flanked by "inhibitory" troughs.

The lack of spontaneous activity of many ICx neurons suggests that ICx neurons may have high thresholds for firing. The high threshold can also lend a mechanism to the elimination of phase ambiguity. At the array-specific ITD, all ICc neurons in an array are activated and may supply adequate excitatory inputs to the postsynaptic ICx neuron to reach the threshold for firing. At other ITDs, not all ICc neurons in the array are excited, and inputs may not be sufficient to activate the postsynaptic ICX neurons. Even if this is the case, the present results show that GABAergic inhibitory interactions across frequency channels are required to eliminate phase ambiguity, as Takahashi and Konishi (1986) hypothesized. One point that turned out to be different from the hypothesis is that inhibition operates at favorable ITDs, and troughs are not locally inhibited when ICx neurons are stimulated with tone.

BMI increased responses to noise at most troughs, whereas troughs adjacent to the primary peak often remained low even after a long period (more than $1 \mathrm{hr}$ ) of BMI administration (Fig. $12 A, B$ ). This observation agrees well with the hypothesis that ICc neurons with a common favorable ITD converge upon the same ICx neurons. If we superimpose ITD curves of these ICc neurons to make a composite ITD curve, the troughs adjacent to the common peak would overlap among these ICc neurons, while other troughs would overlap with peaks of other ICc neurons (Takahashi and Konishi, 1986; Yin et al., 1986). The postsynaptic ICx neurons would therefore receive only small excitatory inputs at the troughs next to the primary peak, and BMI could disinhibit these troughs only by a small amount. This inhibition, however, raised these troughs above the spontaneous level. Because the unfavorable ITDs where these troughs occur may correspond to the horizontal dimension of the inhibitory surround of the receptive field observed in free-field experiments (Knudsen and Konishi, 1978b), we would predict that the application of BMI would eliminate the inhibitory surround.

\section{Temporal discharge patterns of auditory responses}

Neurons in higher nuclei of the time pathway tend to show more phasic responses. The change in temporal pattern occurs progressively along the pathway (Table 2 ). Although rapid adaptation of the responses may well be caused by intrinsic biophysical membrane properties, the present study shows that reduction of GABAergic inhibition changes the discharge patterns from phasic to tonic, suggesting that GABAergic inhibition controls the temporal properties of the auditory responses. One of the advantages of phasic responses over tonic ones may be an increase in the temporal resolution of neuronal computation. It is known that responses of neurons in the magnocellular stream of the primate visual system tend to be more phasic than in the parvocellular streams (Schiller and Malpeli, 1978; Petersen et al., 1988). The magnocellular stream is involved in motion analysis and may require better time resolution than the parvocellular streams (Livingston and Hubel, 1987; DeYoe and Van Fssen, 1988).

\section{GABAergic inhibition in other systems}

Effects of BMI or GABA on maintained discharge rate, rateintensity function, or temporal response characteristics have been examined in the mammalian auditory brainstem (Caspary et al., 1985) and avian auditory forebrain (Müller and Scheich, 1988). These studies, however, have not examined neuronal coding features that are directly related to receptive field properties. Results relevant to the present study are available in other sensory systems.

In the visual cortex, the iontophoretic application of bicu- 
culline to simple cells abolishes the "on" and "off" subdivisions of the receptive field and reduces orientation selectivity (Sillito, 1975, 1984). More complete removal of GABAergic inhibition by a combined use of bicuculline and an inhibitor of GABAergic biosynthesis (Tsumoto et al., 1979) or by BMI (Sillito et al., 1980) completely eliminates orientation selectivity in some simple cells. Generation of orientation selectivity therefore involves intracortical GABAergic inhibition and cannot be explained solely by the convergence of excitatory inputs from afferent neurons as originally proposed (Hubel and Wiesel, 1962). The mechanism for generation of orientation selectivity is, however, still controversial (Ferster and Koch, 1987).

In the somatosensory cortex (Dykes et al., 1984) and thalamus (Hicks et al., 1986) and in the electrosensory lateral-line lobe of weakly electric fish (Shumway and Maler, 1989), BMI altered the spatial and/or temporal properties of the receptive field. These and our present results indicate that inhibitory synaptic interactions operate at some stages for establishment of receptive fields in many sensory modalities, and that some of this inhibition is mediated by GABA.

In conclusion, iontophoretic administration of BMI altered several auditory response properties in 4 successive nuclei of the owl's auditory pathway. BMI decreased the ITD selectivity of ICc core, ICc lateral-shell, and ICX neurons, led to a loss of ICx neurons' ability to signal unique ITDs, and increased response magnitude and altered temporal discharge patterns in all nuclei. GABA, on the other hand, suppressed discharges of these neurons, giving results opposite to those of BMI. The results suggest that GABAergic inhibition plays major and specific roles in the processing of ITD in each nucleus of the time pathway and contributes to delineation of the horizontal dimension of auditory receptive fields.

\section{References}

Andrews PR, Johnston GAR (1979) GABA agonists and antagonists. Biochem Pharmacol 28:2697-2702.

Bloom FE (1974) To spritz or not to spritz: the doubtful value of aimless iontophoresis. Life Sci 14:1819-1834.

Bormann J (1988) Electrophysiology of $\mathrm{GABA}_{A}$ and $\mathrm{GABA}_{\mathrm{B}}$ receptor subtypes. Trends Neurosci 11:112-116.

Brugge JF, Anderson DJ, Aitkin LM (1970) Responses of neurons in the dorsal nucleus of the lateral lemniscus of cat to binaural tonal stimulation. J Neurophysiol 33:441-458.

Carr CE, Konishi M (1988) Axonal delay lines for time measurement in the owl's brainstem. Proc Natl Acad Sci USA 85:8311-8315.

Carr CE, Konishi M (1990) A circuit for detection of interaural time differences in the brainstem of the barn owl. J Neurosci 10:32273246 .

Carr CE, Fujita I, Konishi M (1989) Distribution of GABAergic neurons and terminals in the auditory system of the barn owl. J Comp Neurol 286:190-207.

Caspary DM, Rybak LP, Faingold CL (1985) The effects of inhibitory and excitatory amino-acid neurotransmitters on the response properties of brainstem auditory neurons. In: Auditory biochemistry (Drescher DG, ed), pp 198-226. Springfield: Thomas.

Colburn S, Han Y, Passaro C (1990) Coincidence model of MSO responses. Hearing Res, in press.

DeYoe EA, Van Essen DC (1988) Concurrent processing streams in monkey visual cortex. Trends Neurosci 11:219-226.

Dykes RW, Landry R, Metherate R, Hicks TP (1984) Functional role of GABA in cat primary somatosensory cortex: shaping receptive fields of cortical neurons. J Neurophysiol 52:1066-1093.

Ferster D (1986) Orientation selectivity of synaptic potentials in neurons of cat primary visual cortex. J Neurosci 6:1284-1301.

Ferster D, Koch C (1987) Neuronal connections underlying orientation selectivity in cat visual cortex. Trends Neurosci 10:487-492.

Frank K, Becker MC (1964) Microelectrodes for recording and stim- ulation. In: Physical techniques in biological research, Vol 5, P1 A (Nastuk WL, ed), pp 22-87. New York: Academic.

Fujita I, Konishi M (1988) GABA-mediated inhibition contributes to neuronal selectivity for interaural time difference in the owl's inferior colliculus. Soc Neurosci Abstr 14:1096.

Fujita I, Konishi M (1989a) Inhibitory processes in the formation of the auditory receptive field. In: Neural mechanisms of behaviorProceedings of the 2nd International Congress of Neuroethology (Erbner J, Menzel R, Pflüger H-J, Todt D, eds), p 129. Stuttgart: Georg Thieme.

Fujita I, Konishi M (1989b) Transition from single to multiple frequency channels in the processing of binaural disparity cues in the owl's midbrain. Soc Neurosci Abstr 15:114.

Gardner EP, Constanzos RM (1980) Temporal integration of multiplepoint stimuli in primary somatosensory cortical receptive fields of alert monkeys. J Neurophysiol 43:444-468.

Goldberg JM, Brown PB (1969) Response of binaural neurons of dog superior olivary complex to dichotic tonal stimuli: some physiological mechanisms of sound localization. J Neurophysiol 32:613-636.

Grün S, Aertsen A, Wagner H, Carr C (1990) Sound localization in the barn owl: a quantitative model of binaural interaction in the nucleus laminaris. Soc Neurosci Abstr 16:871.

Hicks TP, Metherate R, Landry P, Dykes RW (1986) Bicucullineinduced alterations of response properties in functionally identified ventroposterior thalamic neurones. Exp Brain Res 63:248-264.

Hubel DH, Wiesel TN (1962) Receptive fields, binocular interaction and functional architecture in the cat's visual cortex. J Physiol (Lond) 160:106-154.

Iversen LL, Bloom FE (1972) Studies of the uptake of ${ }^{3} \mathrm{H}-\mathrm{GABA}$ and $\left[{ }^{3} \mathrm{H}\right]$ glycine in slices and homogenates of rat brain and spinal cord by electron microscopic autoradiography. Brain Res 41:131-143.

Johnston GAR, Beart PM, Curtis DR, Game CJA, McCulloch RM, Maclachlan RM (1972) Bicuculline methochloride as a GABA antagonist. Nature (New Biol) 240:219-220.

Kelly DB, Nottebohm F (1979) Projections of a telencephalic auditory nucleus - field L-in the canary. J Comp Neurol 183:455-470.

Knudsen EI (1984) Synthesis of a neural map of auditory space in the owl. In: Dynamic aspects of neocortical function (Edelman GM, Gall WE, Cowman WM, eds), pp 375-396. New York: Wiley.

Knudsen EI, Konishi M (1978a) Space and frequency are represented separately in auditory midbrain of the owl. J Neurophysiol 41:870884.

Knudsen EI, Konishi M (1978b) Center-surround organization of auditory receptive fields in the owl. Science 202:778-780.

Konishi M (1986) Centrally synthesized maps of sensory space. Trends Neurosci 9:163-168.

Konishi M, Takahashi TT, Wagner H, Sullivan WE, Carr CE (1988) Neurophysiological and anatomical substrates of sound localization in the owl. In: Auditory function-Neurobiological bases of hearing (Edelman GM, Gall WE, Cowan WM, eds), pp 721-745. New York: Wiley.

Kuwada S, Yin TCT, Wickesberg RE (1979) Response of cat inferior colliculus neurons to binaural beat stimuli: possible mechanisms for sound localization. Science 206:586-588.

Livingston MS, Hubel DH (1987) Psychophysical evidence for separate channels for the perception of form, color, movement and depth. J Neurosci 7:3416-3468.

Moiseff A, Konishi M (1981) Neuronal and behavioral sensitivity to binaural time differences in the owl. J Neurosci 1:40-48.

Moiseff A, Konishi M (1983) Binaural characteristics of units in the owl's brainstem auditory pathway: precursors of restricted spatial receptive fields. J Neurosci 3:2553-2562.

Moiseff A, Konishi M (1989a) Binaural disparity cues available to the barn owl for sound localization. J Comp Physiol 164:629-636.

Moiseff A, Konishi M (1989b) Bi-coordinate sound localization by the barn owl. J Comp Physiol 164:637-644.

Müller CM, Scheich H (1988) Contribution of GABAergic inhibition to the response characteristics of auditory units in the avian forebrain. J Neurophysiol 59:1673-1689.

Olsen RW, Ban M, Miller T, Johnston GAR (1975) Chemical instability of the GABA antagonist bicuculline under physiological conditions. Brain Res 98:383-387.

Petersen SE, Miezin FM, Allman JM (1988) Transient and sustained responses in four extrastriate visual areas of the owl monkey. Exp Brain Res 70:55-60. 
Pong SF, Graham LT Jr (1972) $N$-methyl bicuculline, a convulsant more potent than bicuculline. Brain Res 42:486-490.

Rodieck RW (1965) Quantitative analysis of cat retinal ganglion cell response to visual stimuli. Vision Res 5:583-601.

Rose JE, Gross NB, Geisler CD, Hind JE (1966) Some neural mechanisms in the inferior colliculus of the cat which may be relevant to localization of a sound source. J Neurophysiol 29:288-314.

Schiller PH, Malpeli JG (1978) Functional specificity of lateral geniculate nucleus laminae of the rhesus monkey. J Neurophysiol 41:788797.

Shumway CA, Maler L (1989) GABAergic inhibition shapes temporal and spatial response properties of pyramidal cells in the clectrosensory lateral line lobe of gymnotiform fish. J Comp Physiol 164:391-407.

Sillito AM (1975) The contribution of inhibitory mechanisms to the receptive field properties of neurons in the striate cortex of the cat. J Physiol (Lond) 250:305-329.

Sillito AM (1984) Functional considerations of the operation of GABAergic inhibitory processes in the visual cortex. In: Cerebral cortex, Vol 2, Functional properties of cortical cells (Jones EG, Peters A, eds), pp 91-117. New York: Plenum.

Sillito AM, Kemp JA, Milson JA, Berardi N (1980) A re-evaluation of the mechanisms underlying simple cell orientation selectivity. Brain Res 194:517-520.

Sokal RR, Rohlf FJ (1987) Introduction to biostatistics, 2d ed. New York: W. H. Freeman.

Stone TW (1985) Microiontophorcsis and pressure ejection, pp 104 132. Chichester: Wiley.

Sullivan WE, Konishi M (1986) Neural map of interaural phase difference in the owl's brainstem. Proc Natl Acad Sci USA 83:84008404.
Takahashi T, Konishi M (1986) Selectivity for interaural time difference in the owl's midbrain. J Neurosci 6:3413-3422.

Takahashi TT, Konishi M (1988a) Projections of the cochlear nuclei and nucleus laminaris to the inferior colliculus of the barn owl. J Comp Neurol 274:190-211.

Takahashi TT, Konishi M (1988b) Projections of nucleus angularis and nucleus laminaris to the lateral lemniscal nuclear complex of the barn owl. J Comp Neurol 274:212-238.

Takahashi TT, Carr CE, Brecha N, Konishi M (1987) Calcium binding protein-like immunoreactivity labels the terminal field of nucleus laminaris of the barn owl. J Neurosci 7:1843-1856.

Takahashi TT, Wagner H, Konishi M (1989) Role of commissural projections in the representation of bilateral auditory space in the barn owl's inferior colliculus. J Comp Neurol 281:545-554.

Tsumoto T, Eckart W, Creutzfeldt OD (1979) Modification of orientation sensitivity of cat visual cortex neurons by removal of GABAmediated inhibition. Exp Brain Res 34:351-363.

Wagner H, Takahashi TT, Konishi M (1987) Representation of interaural time difference in the central nucleus of the barn owl's inferior colliculus. J Neurosci 7:3105-3116.

Yin TCT, Chan JCK (1988) Neural mechanisms underlying interaural time sensitivity to tones and noise. In: Auditory function-neurobiological hases of hearing (Edelman GM, Gall WE, Cowan WM, eds), pp 385-430. New York: Wiley.

Yin TCT, Chan JCK, Irvine DRF (1986) Effects of interaural time delays of noise stimuli on low-frequency cells in the cat's inferior colliculus. I. Responses to wideband noise. J Neurophysiol 55:280300 . 